\title{
Determinanten des Erfolgs \\ staatlich geförderter Existenzgründungen - eine empirische Untersuchung
}

Philipp G. Sandner, Jörn H. Block, Andreas Lutz

Discussion Paper 2008-5

December 2007

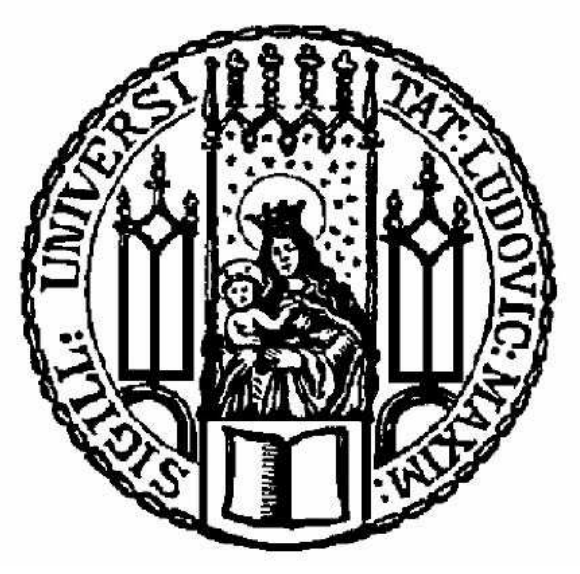

LMU

LUDWIG-MAXIMILIANS-UNIVERSITÄT MÜNCHEN

Munich SCHOOL OF MANAGEMENT

Online at http://epub.ub.uni-muenchen.de/ 


\section{Determinanten des Erfolgs staatlich geförderter Existenzgründungen - eine empirische Untersuchung ${ }^{1}$}

Determinants of Success of Subsidized Start-ups in Germany An Empirical Analysis

Dipl.-Kfm. Philipp G. Sandner

Wissenschaftlicher Mitarbeiter

Institut für Innovationsforschung, Technologiemanagement und Entrepreneurship

Ludwig-Maximilians-Universität München

Kaulbachstraße 45, D-80539 München

Email: psandner@bwl.uni-muenchen.de, Telefon: +49-89-21805809

\section{Dipl.-Kfm. Jörn Hendrich Block}

Wissenschaftlicher Mitarbeiter

Dr. Theo Schöller-Stiftungslehrstuhl für Technologie- und Innovationsmanagement

Technische Universität München

Arcisstraße 21, D-80333 München

Email: block@wi.tum.de, Telefon: +49-89-28925746

\section{Dr. Andreas Lutz \\ Inhaber}
Gruendungszuschuss.de, Existenzgründungs- und Unternehmensberatung
Orleansstrasse 34, D-81667 München,

Email: lutz@gruendungszuschuss.de, Telefon: +49-89-51657980.

\footnotetext{
Wir bedanken uns bei Georg von Graevenitz, Frank Spiegel und Julian Süß für die ausführliche Durchsicht des Manuskripts. Weiterhin bedanken wir uns bei zwei anonymen Gutachtern für die wertvollen Anmerkungen und bei Felizitas Eichinger für die intensive Zusammenarbeit.
} 


\section{Überblick}

- Ein Großteil der Neugründungen in Deutschland ist auf staatliche Förderung von Existenzgründern zurückzuführen. Dieser Anteil hat sich nach Einführung des als „Ich-AG“ bekannten Existenzgründungszuschusses Anfang 2003 stark erhöht. Die Erfolgsfaktorenforschung hat diese Form von Gründungen bislang jedoch wenig untersucht. Im Rahmen einer großzahligen Befragung von geförderten Existenzgründern untersucht dieser Beitrag die Erfolgsdeterminanten solcher geförderter Existenzgründungen.

- Die Ergebnisse der Analyse zeigen, dass der Gewinn aus der Existenzgründung bei älteren Gründern und bei Gründern mit einer langen Phase der Arbeitslosigkeit vor der Gründung seltener beziehungsweise erst später zur Bestreitung des Lebensunterhalts ausreicht. Dies gilt auch für Franchisegründungen und für Gründungen durch Frauen. In Bezug auf Bildungsvariablen, Gründungserfahrung und Teamgründungen im Vergleich zu Einzelgründungen wurde kein Effekt gefunden. Vorhandene Branchenerfahrung wirkt sich hingegen positiv auf den Erfolg der Gründung aus, Gründungs- und Führungserfahrung dagegen nicht.

- Die Implikationen unserer Ergebnisse für die Entrepreneurship-Forschung sowie für die Entrepreneurship-Praxis werden diskutiert. Herausforderungen aus Sicht der Gründungspolitik werden aufgezeigt.

\section{Summary}

- A large number of new ventures in Germany are undertaken due to government aid. With the introduction of the "Ich-AG" or "me-plc" in January 2003, this share has increased strongly. Entrepreneurship research so far has said very little about the success and the determinants of success of these types of start-ups. With this study, we aim to close this gap. We analyze the success and the determinants of success of these new ventures in a large scale survey of founders that have received governmental aid for their start-up.

- Our results show that the income generated by the venture is less likely to cover the costs of living in particular for elderly founders and for those founders that have experienced a long period of unemployment before they started their venture. This finding is also true for new ventures founded by women as well as for franchise start-ups. As concerns formal education, founder experience, and the fact whether the venture is founded by a team or not no significant effects exist. Industry experience, however, has a significant positive effect on the success of the new venture whereas founding and management experience have no effect.

- We discuss the implications of our findings from a perspective of entrepreneurship research as well as from a perspective of governmental start-up policy. 


\section{A. Einleitung}

Ein Großteil der Neugründungen in Deutschland ist auf staatliche Förderung von Existenzgründern zurückzuführen (vgl. Lehnert, 2004; Bergmann/Sternberg, 2007; Niefert/Tchouvakhina, 2006; Sternberg et al., 2007). Der deutsche Staat fördert z.B. seit August 2006 mit dem Instrument Gründungszuschuss Gründungen aus der Arbeitslosigkeit. Staatliche Förderung von Existenzgründungen aus der Arbeitslosigkeit ist allerdings keineswegs neu. Das Instrument des Gründungszuschusses löst lediglich die beiden schon zuvor existierenden Instrumente Überbrückungsgeld und Existenzgründungszuschuss (auch bekannt als „Ich-AG“) ab (vgl. Lutz, 2007 sowie Tabelle 1 für eine detaillierte Beschreibung der einzelnen Förderinstrumente). ${ }^{1}$ Programme dieser Art sind auch in anderen westeuropäischen Staaten wie z.B. Dänemark oder Großbritannien zu finden (vgl. Bendick/Egan, 1987; Meager, 1996; Parker, 2004). Diese Studie untersucht die Erfolgsdeterminanten solcher Gründungen.

Im Rahmen einer großzahligen Befragung von geförderten Existenzgründern $(\mathrm{N}=1.196)$ sind wir folgenden Fragen im Detail nachgegangen:

1) Wer nimmt eine solche Förderung in Anspruch? Wie lassen sich diese Personen hinsichtlich sozio-demographischer Merkmale charakterisieren? Sind die Geförderten mehrheitlich eher der Gruppe der Necessity- oder eher der Gruppe der Opportunity-Gründer ${ }^{2}$ zuzuordnen?

2) Was sind die Erfolgsdeterminanten einer solchen Förderung beziehungsweise unter welchen Umständen ist eine solche Gründung tragfähig? Gibt es geschlechtsspezifische Unterschiede? Sind Teamgründungen erfolgreicher? Wie sind Franchisegründungen zu beurteilen? Welche Rolle spielen Branchen-, Führungs- und Gründungserfahrung bei geförderten Existenzgründern? Gibt es Selektionseffekte?

Neben dem außergewöhnlich großen Datensatz weist unsere Studie eine weitere Besonderheit auf: Um den Erfolg einer Existenzgründung zu beurteilen, verwenden wir nicht eine absolute Größe wie das durch die Gründung erzielte Einkommen oder die Anzahl der geschaffenen Arbeitsplätze, sondern sehen eine Förderung dann als erfolgreich an, wenn der Geförderte angibt, dass er aus dem Unternehmensgewinn heraus seinen Lebensunterhalt bestreiten kann (für eine ausführliche Übersicht der in der Erfolgsfaktorenforschung verwendeten Erfolgsindikatoren, vgl. Schmidt, 2002,). ${ }^{3}$ Unser Ziel ist es, den Referenzpunkt für den Eintritt des Erfolgs an die individuellen Gegebenheiten des jeweiligen Gründers anzupassen. Aus unserer 
Sicht ist eine solche Betrachtung nahe an der politischen Wirklichkeit, da das Verhältnis von Gewinn zu dem zur Lebenshaltung notwendigen Einkommen das zentrale Kriterium ist, nach dem die Tragfähigkeit eines Gründungsvorhabens von den Arbeitsagenturen zu bemessen ist. Im Rahmen des Antrags für eine zu fördernde Gründung ist die Erstellung eines Businessplans erforderlich, in dem Gründungswillige einen kalkulatorischen Unternehmerlohn angeben müssen. Dessen Höhe bemisst sich nach dem bisherigen beziehungsweise alternativ erzielbaren Einkommen sowie nach den Lebenshaltungskosten des Gründers. Im Gesetz zum Gründungszuschuss ( $\$ 57$ (1) SGB III) wird das Ziel der Sicherung des Lebensunterhaltes auch explizit hervorgehoben: „Arbeitnehmer, die durch Aufnahme einer selbständigen, hauptberuflichen Tätigkeit die Arbeitslosigkeit beenden, haben zur Sicherung des Lebensunterhalts und zur sozialen Sicherung [...] Anspruch auf einen Gründungszuschuss“ (Bundesministerium der Justiz, 2007).

Die Erfolgsfaktorenforschung ist integraler Kernbestandteil der Entrepreneurshipforschung. Für eine ausführliche Darstellung sei auf Jacobsen (2006) und Van Praag (2003) verwiesen. Über die Erfolgsfaktoren bei staatlich geförderte Existenzgründungen gibt es bisher jedoch wenig Literatur. Im Folgenden fassen wir kurz einige Ergebnisse der bisherigen Untersuchungen zusammen. Unser Fokus liegt dabei auf den Eigenschaften des Gründers. Mit Daten des Instituts für Arbeitsmarkt und Berufsforschung (IAB) untersuchte Wießner (2000) den Verbleib in der Selbständigkeit von Existenzgründern, die mit Überbrückungsgeld (vgl. Tabelle 1) gefördert wurden. In seiner Stichprobe waren $70 \%$ der Geförderten drei Jahre nach der Gründung noch selbständig. Die von ihm gefundenen Haupteinflussfaktoren für den Verbleib in der Selbständigkeit sind ein niedriges Alter, verheiratet/zusammenlebend gegenüber alleinstehend, die Höhe des Startkapitals und das Vorhandensein von Branchenerfahrung. Thematisch verwandt zu der Studie von Wießner (2000) sind Studien, die Gründungen aus der Arbeitslosigkeit zum Gegenstand haben. Hinz/Jungbauer-Gans (1999) zeigen mit Daten aus der Region München, dass Gründer aus der Arbeitslosigkeit im Vergleich zu anderen Arbeitslosen über eine bessere Humankapitalausstattung verfügen (vgl. auch Reize, 2000), jedoch Probleme im Bereich der Finanzkapitalausstattung existieren. Pfeiffer/Reize (2000) finden auf Basis von Firmendaten heraus, dass Selektionseffekte insbesondere in Ostdeutschland einen großen Teil des (Nicht)Erfolgs erklären und dass von Gründungen aus der Arbeitslosigkeit nur geringe Beschäftigungseffekte ausgehen (vgl. hierzu auch Hinz/Jungbauer-Gans, 1999). Auf Basis von Daten des KfW-Gründungsmonitors der Jahre 2003 und 2004 vergleichen Niefert/Tchouvakhina (2006) die Merkmale von Gründungen aus der Arbeitslosigkeit 
mit denen anderer Gründungen. Im Ergebnis zeigt sich, dass die Mitarbeiteranzahl und der Finanzierungsbedarf von Gründungen aus der Arbeitslosigkeit im Vergleich zu anderen Gründungen unterdurchschnittlich ist und dass überdurchschnittlich häufig im Bereich unternehmensnaher Dienstleistungen gegründet wird (z.B. im Bereich Unternehmens- und Marketingberatung, Webdesign, Softwareentwicklung).

Ziel unserer Untersuchung ist es nicht, den Sinn einer solchen aktiven Arbeitsmarktpolitik zu beurteilen. ${ }^{4}$ Vielmehr wollen wir im Rahmen dieser Untersuchung aufzeigen, inwieweit Instrumente wie der Gründungszuschuss auf individueller Ebene der geförderten Existenzgründer ihr Ziel erreichen und an welchen Stellen gegebenenfalls Verbesserungsbedarf besteht. Aus der Perspektive der Gründungsforschung liegt unser Beitrag darin, dass wir die Erfolgsfaktoren dieser besonderen Gruppe von Gründungen herausarbeiten, empirisch überprüfen und denen in der Literatur der Erfolgsfaktorenforschung verbreiteten Faktoren gegenüberstellen.

Die Ergebnisse unserer empirischen Untersuchung sind interessant für die Gründungsförderung als auch für die Gründungsforschung: Unsere Untersuchung zeigt, dass der Gewinn aus der Existenzgründung bei älteren Gründern und bei Gründern mit einer langen Phase der Arbeitslosigkeit vor der Gründung seltener zur Bestreitung des Lebensunterhalts ausreicht. Dies gilt auch für Franchisegründungen und für Gründungen durch Frauen. Bezüglich des formalen Bildungsabschlusses, Gründungs- sowie Führungserfahrung und Teamgründung im Vergleich zu Einzelgründung konnte kein Effekt nachgewiesen werden. Vorhandene Branchenerfahrung wirkte sich hingegen positiv auf den Erfolg der Gründung aus.

Unser Beitrag ist wie folgt gegliedert: In Abschnitt B betrachten wir die Erfolgsdeterminanten der staatlichen Förderung von Existenzgründern aus einer theoretischen Perspektive. Wir betrachten hierbei die existierende Literatur der Erfolgsfaktorenforschung und stellen Hypothesen auf. In der Auswahl der Hypothesen orientieren wir uns an den Bedürfnissen der Praxis und betrachten nur solche Faktoren, die von Mitarbeitern der Arbeitsagenturen beobachtbar sind beziehungsweise erfragt werden können. Abschnitt $\mathrm{C}$ beschreibt die durchgeführte $\mathrm{Be}-$ fragung, die daraus ermittelten Variablen und die der Auswertung zugrunde liegende Stichprobe. Außerdem werden deskriptive Ergebnisse im Hinblick auf die Bedeutung der einzelnen Erfolgsfaktoren vorgestellt. Abschnitt D beschreibt die angewandten Auswertungsmethoden (Probit-Analysen und Heckman-Modell), stellt die Ergebnisse der multivariaten Analyse dar und geht auf einige Limitationen unserer Studie ein. Abschnitt E diskutiert die Implikationen 
unserer Ergebnisse aus Sicht der Entrepreneurship-Forschung sowie aus Sicht der Entrepreneurship-Praxis. Herausforderungen aus Sicht der Gründungspolitik werden dabei aufgezeigt.

[Tabelle 1]

\section{B. Erfolgsdeterminanten staatlich geförderter Existenzgründungen aus theoreti- scher Perspektive}

In diesem Abschnitt betrachten wir mögliche Erfolgsfaktoren staatlich geförderter Existenzgründungen aus einer theoretischen Perspektive und stellen hierzu Hypothesen auf.

Unsere Studie befasst sich mit Existenzgründern. Existenzgründer können von Unternehmensgründern dahingehend unterschieden werden, dass erstere vor allem eine Arbeitsumgebung für sich selbst schaffen, Unternehmensgründer hingegen idealtypisch ein Unternehmen gründen, das ab einem bestimmten Zeitpunkt nicht mehr von der Gründerperson selbst abhängt (Szyperski, 1999). Damit verbunden ist ein weiterer Unterschied. Im Vergleich zu Existenzgründungen haben Unternehmensgründungen ein höheres Wachstums- und Beschäftigungspotential (Fallgatter, 2002).

Bei der Auswahl der Hypothesen haben wir uns auf Aspekte beschränkt, die eng mit der Person des Gründers zusammenhängen. Zudem haben wir uns an den Bedürfnissen der Praxis orientiert. Alle Variablen, über die wir Hypothesen aufstellen, können von Mitarbeitern der Arbeitsagenturen beobachtet beziehungsweise erfragt werden. Bei der Herleitung der Hypothesen greifen wir die Ergebnisse der bereits existierenden Literatur zur Erfolgsfaktorenforschung auf.

In den ersten beiden Hypothesen betrachten wir den Weg, der zur Existenzgründung geführt hat. Wir argumentieren in Hypothese 1, dass Gründer, die aus dem der Gründung unmittelbar vorangehenden Beschäftigungsverhältnis unfreiwillig durch Kündigung oder BetriebsschlieBung ausgeschieden sind („Necessity-Gründer“), als Existenzgründer anschließend auch weniger erfolgreich sind. ${ }^{5}$ Zwei Begründungen lassen sich hierzu anführen: erstens, bezüglich der Vorgründungsphase sind „Necessity-Gründer“ im Vergleich zu anderen Existenzgründern im Nachteil (zur Bedeutung der Vorgründungsphase, vgl. Jungbauer-Gans/Preisendörfer, 1991, sowie Mellewigt/Witt, 2002). Sie hatten beispielsweise weniger Zeit, ihr berufliches und privates Netzwerk im Hinblick auf die spätere Gründung auszurichten (vgl. Block/Wagner, 2006; Brüderl/Preisendörfer, 1998; Jack/Anderson, 2002). Zweitens sind die 
Opportunitätskosten eines „Necessity-Gründers“ geringer als diejenigen eines Gründers, der von sich aus sein vorheriges Beschäftigungsverhältnis beendet hat. „Necessity-Gründer“ sollten mit einer größeren Wahrscheinlichkeit Existenzgründungen durchführen, die wenig Aussicht auf hohen Gewinn bieten (vgl. Block/Wagner, 2006; Evans/Leighton, 1989; zum Zusammenhang zwischen Opportunitätskosten und Gründungsgeschehen, vgl. Amit et al., 1995). Unsere erste Hypothese ist wie folgt:

H1: Die Wahrscheinlichkeit, dass der Gewinn aus der Existenzgründung zur Bestreitung des Lebensunterhalts ausreicht, ist bei „Necessity-Gründern“ geringer als bei anderen Existenzgründern.

Unsere zweite Hypothese befasst sich mit der Dauer der vorangehenden Arbeitslosigkeit und dem späteren Erfolg als Unternehmer. Hier lässt sich eine ähnliche Argumentation wie im Falle der ersten Hypothese führen. Je länger die Dauer der Arbeitslosigkeit vor der Gründung war, desto länger war der Existenzgründer nicht aktiv ins Berufsleben eingebunden. Er hatte im Vergleich zu anderen Gründern weniger Möglichkeiten, ein für die Gründung hilfreiches soziales Netzwerk aufzubauen beziehungsweise sein vorhandenes Netzwerk kann verloren gehen. Zudem hat er einen schlechteren Zugang zu Informationen, die ihm das Finden einer attraktiven Geschäftsidee erleichtern oder gar erst ermöglichen (vgl. Shane, 2000). Weiterhin gilt: Die Opportunitätskosten, eine wenig Erfolg versprechende Gründung durchzuführen, sinken mit der Anzahl der Monate in Arbeitslosigkeit (vgl. Hinz/Jungbauer-Gans, 1999; Devine/Kiefer, 1993). Selektionseffekte sind ein weiteres Argument für einen negativen $\mathrm{Zu}-$ sammenhang zwischen Dauer der vorangehenden Arbeitslosigkeit und dem Erfolg der Existenzgründung. Diejenigen Gründer, die sich erst nach einer langen Phase der Arbeitslosigkeit zur Gründung entschieden haben, sind tendenziell weniger gut mit wichtigen Unternehmereigenschaften wie z.B. Durchsetzungsfähigkeit oder Kreativität ausgestattet. Unsere zweite Hypothese lautet daher:

H2: Die Wahrscheinlichkeit, dass der Gewinn aus der Existenzgründung zur Bestreitung des Lebensunterhalts ausreicht und die Dauer der vorangehenden Arbeitslosigkeit stehen in einem negativen Zusammenhang.

Die Beweggründe von Frauen für den Weg in die berufliche Selbständigkeit unterscheiden sich von denen von Männern. Die familiäre Situation und die Vereinbarkeit von Beruf und Familie sind für Frauen ein Hauptgrund für den Schritt in die berufliche Selbständigkeit (vgl. 
Carr, 1996; Lauxen-Ulbricht/Leicht, 2005; Parker, 2004, S. 124ff.). Dieser Aspekt spielt bei Gründungen durch Männer eine untergeordnete Rolle. Existenzgründerinnen verfolgen daher in der Regel auch weniger stark Wachstums- und Gewinnmaximierungsziele, sondern wollen vor allem häufig durch eine möglichst hohe Flexibilität in beruflicher Hinsicht die Vereinbarkeit von Beruf und Familie verbessern. Unsere dritte Hypothese lautet daher:

H3: Die Wahrscheinlichkeit, dass der Gewinn aus der Existenzgründung zur Bestreitung des Lebensunterhalts ausreicht, ist bei Existenzgründern höher als bei Existenzgründerinnen.

Der Zusammenhang zwischen der Humankapitalausstattung eines Gründers und dem späteren Erfolg der Gründung ist in der Gründungsforschung bereits mehrfach untersucht worden (vgl. z.B. Davidsson/Honig, 2003; zum Begriff des Humankapitals vgl. Becker, 1964). Im Rahmen dieser Studie betrachten wir drei Aspekte der Humankapitalausstattung eines Existenzgründers näher: seine Gründungserfahrung, seine Führungserfahrung und seine Branchenerfahrung. Generell vermuten wir einen positiven Zusammenhang zwischen der Humankapitalausstattung eines Gründers und dem Erfolg der Gründung. In Bezug auf die Wirkung der von uns betrachteten Humankapitalvariablen lassen sich im Einzelnen folgende Begründungen anführen: Gründungserfahrung beschreibt die Tatsache, dass ein Gründer bereits einmal zuvor ein Unternehmen gegründet hat beziehungsweise an einer Gründung mitgewirkt hat. Viele Fähigkeiten, die zur erfolgreichen Umsetzung eines Geschäftsplans nötig sind, lassen sich durch „learning by doing“ am Besten erlernen (vgl. Brüderl et al., 1992; Jovanovic, 1982). Duchesneau/Gartner (1990) führen in diesem Zusammenhang z.B. an, dass das Sammeln von Informationen, die Trennung wichtiger von unwichtiger Informationen und das damit verbundene Treffen von effektiven Entscheidungen schwer erlernt werden kann ohne tatsächlich unternehmerisch tätig zu sein. Gründer mit Gründungserfahrung haben einen Teil dieses Lernprozesses bereits hinter sich und sind somit im Vorteil gegenüber anderen Gründern. Unsere Hypothese ist daher:

H4a: Die Wahrscheinlichkeit, dass der Gewinn aus der Existenzgründung zur Bestreitung des Lebensunterhalts ausreicht, und das Vorhandensein von Gründungserfahrung seitens des Existenzgründers stehen in einem positiven Zusammenhang.

Führungserfahrung bezieht sich auf die Erfahrung des Gründers in Bezug auf das Management und das Anleiten von Mitarbeitern. Für eine junge Unternehmung ist Führungserfahrung 
besonders wichtig, da noch keine Verhaltens- und Organisationsmuster bestehen (Szyperski/Nathusius 1999, S. 40). Insbesondere in der Gewinnung von qualifiziertem Personal und im Anleiten dieses Personals für die Zwecke des Unternehmens kann Führungserfahrung zu einem kritischen Erfolgsfaktor werden (Brüderl et al., 1992). Ein weiteres Argument spricht für einen positiven Zusammenhang zwischen Führungserfahrung und Gründungserfolg: Gründer mit Führungserfahrung haben häufig bereits mehrere Karrierestationen bei ihrem alten Arbeitgeber durchlaufen, was ihnen einen guten Zugang zu einer großen Bandbreite an Informationen verschafft. Auch sollten sie über ein breites berufliches Netzwerk verfügen. Beides kann bezüglich der Entdeckung und bezüglich der Umsetzung einer Geschäftsidee von großem Vorteil sein (vgl. Jack/Anderson, 2002; Romanelli/Schoonhoven, 2001). Wir formulieren daher folgende Hypothese:

H4b: Die Wahrscheinlichkeit, dass der Gewinn aus der Existenzgründung zur Bestreitung des Lebensunterhalts ausreicht, und das Vorhandensein von Führungserfahrung seitens des Existenzgründers stehen in einem positiven Zusammenhang.

Ein hohes Maß an Branchenerfahrung erleichtert es dem potentiellen Gründer, lukrative Geschäftsmöglichkeiten zu erkennen und diese dann auf ihre Machbarkeit hin zu überprüfen (vgl. Shane, 2003). Die Unternehmensgründung wird außerdem stärker an den Kundenbedürfnissen orientiert sein, was die Erfolgschancen positiv beeinflusst (vgl. Picot et al., 1989). Ist eine lukrative Geschäftsmöglichkeit gefunden, so hilft Branchenerfahrung maßgeblich bei der Bewertung und Beschaffung von Ressourcen, die zur konsequenten Umsetzung der entdeckten Geschäftsidee benötigt werden (z.B. Personal- oder Finanzressourcen). Unsere Hypothese lautet:

H4c: Die Wahrscheinlichkeit, dass der Gewinn aus der Existenzgründung zur Bestreitung des Lebensunterhalts ausreicht und das Vorhandensein von Branchenerfahrung seitens des Existenzgründers stehen in einem positiven Zusammenhang.

Im Folgenden gehen wir kurz auf zwei Aspekte näher ein, über die wir a priori keine eindeutigen Hypothesen formulieren können, die aber-wie sich zeigen wird—in den Ergebnissen unserer Studie von zentraler Bedeutung sind.

Hinsichtlich des Zusammenhangs zwischen dem Alter des Gründers und dem Gründungserfolg wird in der Mehrzahl der bisherigen Studien der Erfolgsfaktorenforschung eine umgekehrt U-förmige Beziehung gefunden (vgl. z.B. Block/Sandner, 2007; Mincer, 1974; 
van Praag, 2003; Schwarz et al., 2005). Die theoretische Begründung hierfür ist: Auf der einen Seite erhöhen sich mit zunehmendem Alter die berufliche und allgemeine Lebenserfahrung (vgl. Shane, 2003), die Zahl der beruflichen und privaten Kontakte (vgl. Jack/Anderson, 2002) sowie die Möglichkeit, eigene finanzielle Ersparnisse als Startkapital zu verwenden. Auf der anderen Seite sinkt die Risikobereitschaft aufgrund etablierter Familienverhältnisse sowie der Angst, bereits erreichtes Vermögen wieder zu verlieren (vgl. Kahneman et al., 1991). In unserem Fall lässt sich jedoch ein U-förmiger Zusammenhang nicht ex ante vermuten. Die Wahrscheinlichkeit, dass der Gewinn aus der Existenzgründung zur Bestreitung des Lebensunterhalts ausreicht, ist neben den oben genannten Faktoren auch noch von dem individuellen Lebensstil des Existenzgründers abhängig. Es lässt sich vermuten, dass aufgrund von Familie und erreichtem Lebensstandard ein älterer Gründer höhere Lebenshaltungskosten hat als ein jüngerer Gründer. Darüber, ob dieser Aspekt jedoch schwerer wiegt als die gesteigerte Produktivität aufgrund von mehr Erfahrung und einer höheren Anzahl sozialer Kontakte, lässt sich nur spekulieren.

Über den Einfluss von Franchisegründungen im Vergleich zu anderen Gründungsformen formulieren wir ex ante ebenfalls keine Hypothese. Die Wirkungsrichtung ist aus theoretischer Perspektive nicht eindeutig. Einerseits ist eine Franchisegründung aufgrund des bereits erprobten Geschäftsmodells mit geringerem Risiko verbunden und sollte somit dem Franchisenehmer ein Mindesteinkommen garantieren. Durch opportunistisches Trittbrettfahrerverhalten gegenüber dem Franchisegeber könnte er dieses Einkommen auch noch steigern (vgl. Albach, 2005, S. 16f.; Ehrmann, 2007). Andererseits könnte der Franchisegeber Wachstumsund Gewinnziele verfolgen, die zu Lasten des Franchisenehmers gehen oder seinen Wissensvorsprung über das Franchisesystem ausnutzen und vom Franchisenehmer eine unangemessen hohe Franchisegebühr verlangen. Aus der Perspektive eines Franchisenehmers hängt die Attraktivität eines Franchisevertrags von Faktoren wie dem Erfolg des Geschäftsmodells, der ihm gewährten Exklusivität innerhalb eines bestimmten Verkaufsgebietes und der Höhe der an den Franchisegeber abzuführenden Franchisegebühr ab (zur Ökonomik von Franchiseverträgen, vgl. Mathewson/Winter, 1985).

\section{Forschungsdesign und deskriptive Ergebnisse}

In diesem Abschnitt beschreiben wir unser Forschungsdesign und die der Untersuchung zugrunde liegende Stichprobe. Außerdem untersuchen wir anhand univariater Statistiken durch welche Merkmale sich erfolgreiche von nicht erfolgreichen Gründungen unterscheiden. 
Als Forschungsdesign wurde die Erhebung mittels Fragebogen gewählt. Der Fragebogen wurde im November 2006 entwickelt und enthielt Fragen zu den Eigenschaften des Existenzgründers sowie den Merkmalen der Gründung. Der Fragebogen wurde nach der Konzeption mehreren Pre-Tests mit Existenzgründern und Gründungsberatern unterzogen. In Kooperation mit der Gründungsberatung ,gruendungszuschuss.de“"wurde dann eine Onlineversion des Fragebogens erstellt. Mit Hilfe unseres Kooperationspartners wurden dann anschließend insgesamt 33.695 Personen per E-Mail angeschrieben und auf den Fragebogen hingewiesen. ${ }^{6}$ Innerhalb des Befragungszeitraums vom 7.12.2006 bis zum 18.12.2006 erhielten wir insgesamt 3.295 Antworten, was einer Antwortquote von 9,78\% entspricht. Nach einer Bereinigung des Datensatzes um 475 Personen, die vor dem Jahr 2002 gegründet haben, 705 Personen, die keine staatliche Förderung in Anspruch genommen haben sowie Beobachtungen mit fehlenden Werten bei den uns interessierenden Variablen (919 Personen) blieben 1.196 Fragebögen zur Auswertung übrig. Von diesen 1.196 Personen waren 90 Personen zum Zeitpunkt der Befragung bereits nicht mehr selbstständig tätig. Tabelle 2 gibt einen Überblick über die in der Analyse verwendeten Variablen und ihren jeweiligen Ausprägungen. Tabelle 3 beschreibt die Stichprobe bezüglich dieser Variablen, Tabelle 4 vergleicht erfolgreiche und nicht erfolgreiche Gründer anhand univariater Statistiken.

\section{[Tabelle 2] [Tabelle 3] [Tabelle 4]}

Für 65\% der Existenzgründer reichte der Gewinn aus der selbständigen Tätigkeit zum Zeitpunkt der Befragung bereits zur Bestreitung des Lebensunterhalts aus (ERFOLG). Dies ist bemerkenswert hoch, da sich die Mehrzahl der Gründungen noch im ersten Jahr der Gründung befinden. Die Mehrzahl der Gründer ist mit der Gründung zufrieden (Mittelwert=3,98; 1=,,sehr unzufrieden“, 5=,,sehr zufrieden“"). Erfolgreiche Gründer sind dabei zufriedener als nicht erfolgreiche (4,21 vs. 3,55 mit p<0,001). 97\% der Befragten würden die Gründung wiederholen. Hinsichtlich der Förderung haben $8 \%$ der Befragten Existenzgründungszuschuss (Ich-AG), 71\% Überbrückungsgeld, 19\% Gründungszuschuss und 2\% Einstiegsgeld erhalten.

68\% der Befragten gaben an, dass sie ihr letztes Arbeitsverhältnis vor Eintritt in die Selbständigkeit unfreiwillig verlassen haben (NECESSITY). Interessant ist hierbei, dass ein signifikanter Unterschied zwischen erfolgreichen und nicht erfolgreichen Gründern dahingehend besteht, dass nicht erfolgreiche Gründer ihren Arbeitsplatz häufiger unfreiwillig verlassen haben (77\% vs. 63\% mit p<0,001). Dies kann ein Hinweis für das Zutreffen von Hypothese 1 sein, auch wenn es sich hier zunächst nur um einen univariaten Test handelt. Analog zu der 
Variable NECESSITY ist die Dauer der Arbeitslosigkeit vor dem Eintritt in die Selbständigkeit bei erfolgreichen Gründern signifikant geringer (3,6 Monate vs. 6,8 Monate mit p<0,001), was auf ein Zutreffen von Hypothese 2 hindeutet.

Bezüglich gründungsspezifischer Merkmale gilt: 30\% der Gründer haben für ihre Existenzgründung Fremdkapital aufgenommen und 15\% der Befragten haben im Team gegründet. Zwischen erfolgreichen und nicht erfolgreichen Existenzgründern gibt es bezüglich beider Variablen jedoch keine signifikanten Unterschiede. 2\% der Befragten haben ein Franchisekonzept bei der Gründung verfolgt. Dabei sind diese Gründer signifikant häufiger in der Gruppe der nicht erfolgreichen Gründer zu finden ( $2 \%$ vs. $4 \%$ mit $\mathrm{p}=0,023)$.

Demographische Variablen in unserer Befragung umfassen personenspezifische Eigenschaften wie Geschlecht, Alter und Nationalität. Mit 69\% der Befragten sind mehr als zwei Drittel der Existenzgründer männlich (GESCHLECHT). Dabei sind Männer in der Gruppe der erfolgreichen Unternehmer signifikant überrepräsentiert (75\% vs. 57\% mit $\mathrm{p}<0,001$ ), was einen Hinweis für das Zutreffen von Hypothese 3 darstellt. Das Durchschnittsalter der befragten Gründer ist 41 Jahre. Erfolgreiche Gründer sind signifikant jünger (40,1 vs. 42,6 Jahre mit $\mathrm{p}<0,001)$. Bezüglich der Bildung haben wir nach dem höchsten erreichten Bildungsabschluss gefragt. 7\% der befragten Existenzgründer haben einen Realschulabschluss, 10\% Abitur, 21\% eine Berufsausbildung, 4\% eine Meisterprüfung, 47\% einen Studienabschluss und 4\% eine Promotion. In der Gruppe der erfolgreichen Gründer sind mehr Gründer mit Studienabschluss ( $49 \%$ vs. $43 \%$ mit $\mathrm{p}=0,049)$.

Neben der Bildung haben wir auch die Erfahrung des Gründers mit einbezogen. Dabei haben wir mit Branchen-, Gründungs- und Führungserfahrung drei Facetten der Erfahrung abgebildet (Brüderl et al. 1998). Branchenerfahrung misst, ob der Befragte sein Unternehmen in einer Branche gegründet hat, in der er zuvor beruflich tätig war. Dies wurde von $76 \%$ der Befragten bejaht. Gründungserfahrung hat ein Gründer, wenn er sich zuvor bereits mindestens einmal hauptberuflich selbständig gemacht hat (33\%). Weiterhin wollten wir wissen, ob der Gründer vor seinem Schritt in die Selbständigkeit bereits eine Anstellung mit Führungserfahrung inne hatte $(65 \%)$. Signifikante Unterschiede zwischen erfolgreichen und nicht erfolgreichen Existenzgründern zeigen sich nur bezüglich Branchenerfahrung ( $81 \%$ vs. $65 \%$ mit $\mathrm{p}<0,001$ ), was auf ein Zutreffen von Hypothese $4 c$ hindeutet. 
Branchenzugehörigkeit, regionale Variablen und Gründungsjahr wurden erfragt, um in der multivariaten Analyse auf mögliche Einflüsse dieser Variablen kontrollieren zu können. Die univariaten Ergebnisse sind in den Tabellen 3 und 4 dargestellt.

\section{Ergebnisse der multivariaten Analyse}

Im Rahmen der multivariaten Analyse schätzen wir zwei Typen von Modellen: ein einfaches Probit-Modell (Wooldridge, 2002, S. 530-354 ) und ein zweistufiges Heckman-Probit-Modell (Heckman, 1976). Heckman-Modelle werden verwendet um auf Selektionseffekte zu kontrollieren beziehungsweise zu testen, ob diese überhaupt vorliegen. Unser Heckman-Modell besteht aus zwei Probit-Schätzungen. Abhängige Variable in der 1. Stufe ist die Tatsache, ob der Befragte zum Zeitpunkt der Befragung noch selbständig ist. Abhängige Variable in der zweiten Stufe ist die Einschätzung des Befragten, ob der Gewinn aus seiner Existenzgründung zur Bestreitung seines Lebensunterhalts ausreicht. ${ }^{7}$ Die Wahrscheinlichkeit, dass der Gewinn aus der Existenzgründung zur Bestreitung des Lebensunterhalts von Existenzgründer $i$ ausreicht, wird durch die folgende latente Funktion beschrieben (2. Stufe des Heckman-Modells)

$$
y_{i}^{*}=\mathbf{X}_{i} \beta+e_{1 i}
$$

wobei $\mathrm{X}_{\mathrm{i}}$ einen Vektor an erklärenden Variablen, $\beta$ den zu schätzenden Parametervektor und $\mathrm{e}_{1 \mathrm{i}}$ eine normalverteilte Zufallsvariable mit Mittelwert 0 und Standardabweichung 1 darstellt. Der Zusammenhang zwischen $y_{\mathrm{i}}{ }^{*}$ und der Tatsache, ob der Gewinn zur Bestreitung des Lebensunterhalts ausreicht, $y_{\mathrm{i}}$, ist nichtlinear und folgt der Standardnormalverteilung. Es gilt:

$$
y_{i}= \begin{cases}1 & \text { wenn } y_{i}^{*} \geq 0 \\ 0 & \text { sonst }\end{cases}
$$

$\mathrm{y}_{\mathrm{i}}$ kann jedoch nur beobachtet werden, wenn der Existenzgründer zum Zeitpunkt der Befragung noch selbständig ist. Die Selektionsgleichung (1. Stufe des Heckman-Modells) lautet:

$$
\mathrm{Z}_{i} \gamma+e_{2 i}>0
$$

wobei $\mathrm{Z}_{\mathrm{i}}$ einen Vektor an Variablen, die einen Einfluss auf das Überleben der Existenzgründung bis zum Zeitpunkt der Befragung haben, $\gamma$ einen zu schätzenden Parametervektor und $e_{2 i}$ eine normalverteilte Zufallsvariable mit Mittelwert 0 und Standardabweichung 1 darstellt. Es wird angenommen das die Fehler $\mathrm{e}_{1 \mathrm{i}}$ und $\mathrm{e}_{2 \mathrm{i}}$ einer gemeinsamen Verteilung folgen und die 
Korrelation $\rho$ aufweisen. Ist $\rho \neq 0$, so sind (1) und (3) voneinander abhängig und ein einstufiges Probit-Modell würde verzerrte Schätzer aufweisen. Ein Test darauf, ob $\rho$ signifikant verschieden von 0 ist, kann somit als ein Indikator für das Vorliegen von Selektion dienen.

Da es sich sowohl beim normalen Probit-Modell als auch beim zweistufigen Heckman-ProbitModell um nichtlineare Regressionsmodelle handelt, können die Koeffizienten nicht direkt interpretiert werden. Nur Signifikanz und Vorzeichen der Koeffizienten sind direkt interpretierbar. Zur besseren Interpretation haben wir daher zusätzlich marginale Effekte berechnet. Die Ergebnisse der durchgeführten Regressionen sind in den Tabellen 6 und 7 abgebildet. Im Rahmen der normalen Probit-Analyse schätzen wir drei Modelle mit unterschiedlichen Spezifikationen der Variable ALTER. Tabelle gibt die Korrelationen zwischen den unabhängigen Variablen und die Varianzinflationsfaktoren wieder. Multikollinearität stellt bei unseren Regressionsmodellen kein großes Problem dar, was die niedrigen Varianzinflationsfaktoren der jeweiligen unabhängigen Variablen zeigen. Außerdem gibt es im Vergleich zwischen univariater und multivariater Analyse bei keiner Variable einen Vorzeichenwechsel.

\section{[Tabelle 5] [Tabelle 6] [Tabelle 7]}

Im Folgenden gehen wir auf die Ergebnisse der Probit-Analyse ein: In Hypothese 1 vermuten wir, dass die Wahrscheinlichkeit, dass der Gewinn zur Bestreitung des Lebensunterhalts ausreicht, bei Necessity-Gründern geringer ist als bei Selbständigen, die von sich aus gekündigt haben („Opportunity-Gründer“). Diese Hypothese kann nicht bestätigt werden. Der Erfolg in der Selbständigkeit scheint unabhängig von der Tatsache zu sein, ob der Existenzgründer der Gruppe der Necessity-Gründer zuzurechnen ist oder nicht $(\beta=-0,091$ mit $\mathrm{p}>0,1$; Modell I). Nach Hypothese 2 stehen die Dauer der vorangehenden Arbeitslosigkeit und der Erfolg in einem negativen Zusammenhang. Die Richtigkeit dieser Hypothese kann bestätigt werden ( $\beta=0,053$ mit $p<0,001$; Modell I). Die Variablen NECESSITY und ARBEITSLOSIGKEIT, die beide über die Vorgründungsphase informieren, sind miteinander korreliert $(r=0,37$ mit $\mathrm{p}<0,05)$. In der multivariaten Analyse hat aber nur die Variable ARBEITSLOSIGKEIT einen signifikanten Einfluss. Die Dauer der Arbeitslosigkeit vor der Gründung scheint daher wichtiger zu sein als die Tatsache, ob der Gründer sein vorheriges Angestelltenverhältnis unfreiwillig verlassen hat oder nicht. Hypothese 3 postuliert, dass die Erfolgswahrscheinlichkeit bei Existenzgründern höher ist als bei Existenzgründerinnen. Diese Hypothese kann bestätigt werden. Die Variable GESCHLECHT hat einen positiv signifikanten Einfluss auf den Erfolg ( $\beta=0,463$ mit $p<0,001$, Modell I). Bzgl. der Hypothesen über den Zusammenhang zwischen 
der Humankapitalausstattung eines Gründers und dem Erfolg der Gründung, finden wir lediglich Evidenz für das Zutreffen von Hypothese 4c. Das Vorhandensein von Branchenerfahrung seitens des Existenzgründers steht in einem positiven Zusammenhang mit der Erfolgswahrscheinlichkeit ( $\beta=0,317$ mit $p<0,01$, Modell I). Für ein Zutreffen der Hypothesen 4a und 4b über eine positive Beziehung zwischen Gründungserfahrung beziehungsweise Führungserfahrung und Gründungserfolg finden wir keine Hinweise.

Wir finden in unserer Studie weitere interessante Zusammenhänge, für die wir ex ante keine Hypothesen aufgestellt haben. So ist die Erfolgswahrscheinlichkeit bei einer Franchisegründung geringer als bei anderen Gründungen $(\beta=-0,704$ mit $p<0,01$; Modell I). Weiterhin erstaunt der Effekt der Variable ALTER. Modell I zeigt einen negativen linearen Zusammenhang zwischen Alter und Erfolg $(\beta=-0,021$ mit $p<0,001)$. Ein quadratischer Zusammenhang besteht hingegen nicht (vgl. Modell II). Um den Zusammenhang zwischen Alter und Erfolg genauer angeben zu können, haben wir in Modell III die Variable Alter in mehrere Dummyvariablen aufgeteilt und diese Variablen dann anschließend ins Modell genommen. Es zeigt sich, dass höhere Altersklassen eine signifikant niedrigere Erfolgswahrscheinlichkeit als die nicht in das Modell aufgenommene niedrigste Altersklasse aufweisen. Die Ergebnisse aus Modell I werden somit bestätigt. Ein negativer linearer Zusammenhang zwischen Alter und Erfolgswahrscheinlichkeit steht jedoch nicht im Einklang mit der Mehrzahl der Studien der Erfolgsfaktorenforschung (vgl. z.B. Mincer, 1974; van Praag, 2003; Schwarz et al., 2005).

F-Tests auf gemeinsame Signifikanz zeigen, dass Branchenvariablen nur einen schwach signifikanten Einfluss auf den Erfolg haben $(p<0,1)$. Regionale Variablen haben dagegen keinen signifikanten Einfluß (p>0,1). Ebenfalls haben Bildungsvariablen keinen signifikanten Einfluss ( $p>0,1)$. Letzteres erstaunt, da wir bei einem univariaten Vergleich zumindest bei einer Bildungsvariablen einen signifikanten Unterschied gefunden haben (vgl. Tabelle 4).

In die 1. Stufe des Heckman-Modells haben wir neben den bereits in die einfache ProbitAnalyse aufgenommenen Variablen noch zusätzlich die beiden Variablen BUSINESSPLANUMFANG und BERATUNGSUNTERSTÜTZUNG aufgenommen. Die 2. Stufe des Heckman-Modells entspricht Modell I der einfachen Probit-Analyse. Die Ergebnisse des Heckman-Modells bestätigen unsere Ergebnisse aus des einfachen Probit-Modell (vgl. Tabelle 7). Obwohl Selektion vorliegt ( $\rho$ ist schwach signifikant verschieden von 0 ), verändern sich die Vorzeichen und die Signifikanz der unabhängigen Variablen nur geringfügig, was auf eine Robustheit unserer Ergebnisse hindeutet. Interessant ist, dass die Variable BUSINESS- 
PLANUMFANG positiv mit der Selektionsvariable zusammenhängt $(\beta=0,089$ mit $p<0,05)$. Der Umfang des Businessplans scheint ein guter Indikator für das (kurzfristige) Überleben einer Gründung zu sein.

Unsere Studie hat einige Limitationen. Sie könnte dahingehend verzerrt sein, dass erfolgreiche Gründer mit einer höheren Wahrscheinlichkeit unserem Aufruf zur Beantwortung des Fragebogens gefolgt sind. Mit den uns zur Verfügung stehenden Daten können wir diese Einschränkung jedoch nicht aufheben. Die Ergebnisse unseres Heckman-Modells deuten jedoch darauf hin, dass die von unseren Variablen ausgehenden Effekte robust sind. Aussagen über die langfristige Tragfähigkeit der von uns untersuchten Existenzgründungen lassen sich mit unserem Datensatz schwer treffen. Von uns als nicht erfolgreich einklassifizierte Gründungen können im Zeitverlauf noch zu erfolgreichen Gründungen werden und umgekehrt. Außerdem könnte es sich bei den nicht erfolgreichen Gründungen auch um Gründungen mit einem überdurchschnittlich hohen Investitionsbedarf in der Startphase handeln Aus diesen und anderen Gründen wäre ein Paneldatensatz wünschenswert. Durch eine mehrfache Befragung desselben Existenzgründers könnte dessen Erfolg im Zeitablauf beobachtet werden und so dessen Entwicklung besser analysiert werden (Ruspini, 2002). Damit könnte z.B. präziser ermittelt werden, ab welchem Zeitpunkt seit Eintritt in die Selbständigkeit der Gewinn aus der Existenzgründung zur Bestreitung des Lebensunterhalts ausreicht.

\section{E. Implikationen}

In diesem Abschnitt werden die Implikationen unserer Ergebnisse für die EntrepreneurshipForschung und -Praxis dargestellt. Die Praxis umfasst hier insbesondere die Akteure der Gründungspolitik sowie die Gründungsberater und die kreditgebenden Banken.

\section{Implikationen für die Entrepreneurship-Forschung}

An Implikationen für die Entrepreneurship-Forschung sind vier Aspekte besonders hervorzuheben: Die besondere Rolle der Variable ALTER, der starke Einfluss der Variable GESCHLECHT, die große Bedeutung der Vorgründungsphase und, ganz allgemein, die Unterschiede in den Erfolgsfaktoren zwischen der Gruppe der geförderten Existenzgründer zu anderen Gruppen von Existenzgründern.

- Der Koeffizient der Variable ALTER ist in unserer Analyse negativ. Je älter der Existenzgründer ist, desto weniger wahrscheinlich ist es, dass der Gewinn aus der Existenzgrün- 
dung zur Bestreitung des Lebensunterhalts ausreicht. Ein umgekehrt U-förmiger Zusammenhang, der normalerweise postuliert wird, wurde nicht gefunden (vgl. auch Tabelle 6, Modell II). Der Grund für dieses, von der Mehrzahl der Studien abweichendes Ergebnis liegt in der Wahl der abhängigen Variable. Entsprechend der Begründung des Gesetzgebers im Gesetz zum Gründungszuschuss (§ 57 (1) SGB 3) wird eine (geförderte) Existenzgründung von uns dann als erfolgreich angesehen, wenn der Gewinn aus der Existenzgründung zur Bestreitung des Lebensunterhalts ausreicht. In den meisten Studien im Bereich der Erfolgsfaktorenforschung wird der Erfolg hingegen unabhängig von den individuellen Bedürfnissen des jeweiligen Existenzgründers betrachtet. Der gefundene negative Zusammenhang in unserer Studie lässt sich dadurch erklären, dass aufgrund von Familie und erreichtem Lebensstandard ältere Gründer höhere Lebenshaltungskosten haben als jüngere Gründer. In unserer, zunehmend älter werdenden Gesellschaft wird dieser Aspekt an Bedeutung gewinnen. Aus unserer Sicht besteht daher Forschungsbedarf im Bereich „Gründungen durch ältere Menschen“.

- Wie in den meisten anderen Studien auch, zeigte sich in unserer Untersuchung, dass Gründerinnen im Durchschnitt weniger erfolgreich sind als Gründer (vgl. Tabelle 6). Aus der Tatsache, dass dieses Ergebnis auch nach Berücksichtigung von Branchenerfahrung, Bildung und Alter Bestand hat, folgern wir, dass Gründungen von Frauen stärker vom Motiv der Vereinbarkeit von Beruf und Familie geleitet sind als Gründungen von Männern (vgl. Carr, 1996; Lauxen-Ulbricht/Leicht, 2002). Monetäre Erfolgsgrößen scheinen bei Gründerinnen einen geringeren Stellenwert zu besitzen als bei Gründern.

- Es zeigte sich in unserer Studie auch, dass die Vorgründungsphase beziehungsweise der Zugang zu relevanten Ressourcen in dieser Phase von entscheidender Bedeutung für den späteren Erfolg der Existenzgründung ist. Sowohl Gründer mit Branchenerfahrung als auch solche mit einer geringen Dauer der Arbeitslosigkeit vor der Existenzgründung waren im Durchschnitt erfolgreicher als andere Gründer. Der Grund hierfür mag darin liegen, dass solche Gründer einen besseren Zugang zu relevanten, schwierig zu beschaffenden Informationen (vgl. Shane, 2000) sowie zu hilfreichen Netzwerken haben (vgl. Jack/Anderson, 2002). Unser Ergebnis steht im Einklang mit einer Theorie der Pfadabhängigkeit, wonach die Evolution eines Unternehmens (hier: eines neu gegründeten Unternehmens) stark durch Pfadabhängigkeit bestimmt wird (vgl. Mellewigt/Witt, 2002; Nelson/Winter, 1982).

- Neben dem Alter des Gründers zeigte sich auch bzgl. der Variable TEAM ein Unterschied. Im Gegensatz zu anderen Untersuchungen (z.B. Picot et al., 1989) waren in unse- 
rer Studie Teamgründungen nicht erfolgreicher als andere Einzelgründungen (vgl. Tabelle 6). Wir interpretieren dieses Ergebnis dahingehend, dass die möglichen Vorteile einer Teamgründung (z.B. Ergänzung von Know-How, größere finanzielle Ressourcen) nicht für alle Formen von Gründungen gleichermaßen relevant sind. Im Vergleich zu beispielsweise innovativen Technologiegründungen sind die Vorteile einer Teamgründung für die Gruppe der geförderten Existenzgründungen weniger bedeutend. Gründungen aus der Arbeitslosigkeit sind typischerweise weniger kapitalintensiv (vgl. Niefert/Tchouvakhina, 2006) und mit weniger technischem Know-How verbunden als andere Gründungen. ${ }^{8}$

\section{Implikationen für die Entrepreneurship-Praxis}

Die Implikationen für die Entrepreneurship-Praxis unterteilen wir in die zwei Kategorien „Eigenschaften von geförderten Existenzgründungen“ und „Erfolgsfaktoren von geförderten Existenzgründungen“.

- In unserer Studie zeigt sich, dass die geförderten Existenzgründer gut ausgebildet sind (mehr als 50\% haben einen Studienabschluß oder eine Promotion) und mehrheitlich über Branchen- und Führungserfahrung verfügen (76\% beziehungsweise 65\%). Immerhin ein Drittel der Gründer hat zuvor bereits einmal ein Unternehmen gegründet. Der Anteil der Gründungen, deren Gewinn zur Bestreitung des Lebensunterhalts ausreicht, ist mit 65\% hoch. Zudem geben mehr als 97\% der Gründer an, dass sie den Schritt in die Selbständigkeit retrospektiv betrachtet nochmals wagen würden. Die Zufriedenheit der Gründer lag im Durchschnitt bei 3,98 (1=sehr unzufrieden; 5=sehr zufrieden). Obwohl diese Zahlen mit Vorsicht zu interpretieren sind (vgl. Limitationen der Studie in Abschnitt D), lässt sich aus unseren Ergebnissen eine positive Grundtendenz ablesen, die auch von anderen Studien bestätigt wird (vgl. Caliendo et al., 2006; Caliendo/Steiner, 2007).

- Die von uns gefundenen Ergebnisse zu den Erfolgsfaktoren haben Implikationen für die Gründungspolitik, für Gründungsberater und für kreditgebende Banken. Für diese drei Gruppen ist z.B. interessant, dass Franchisegründungen im Durchschnitt weniger erfolgreich sind als andere Gründungen und dass Teamgründungen nicht erfolgreicher sind als Einzelgründungen. Bei der Beurteilung der Förderungswürdigkeit beziehungsweise der Beurteilung der Erfolgsaussichten sollte ein besonderes Augenmerk auf die Branchenerfahrung des Gründers gelegt werden. Der formale Bildungsabschluss ist hingegen von geringer Bedeutung. Bei geförderten Existenzgründungen durch Frauen reicht der Gewinn seltener zur Bestreitung des Lebensunterhalts, was sich dadurch erklärt, dass Frauen we- 
niger aus monetären Motiven heraus gründen als Männer. Für die Gründungspolitik weisen unsere Ergebnisse auf zwei zentrale Herausforderungen hin: Erstens hängen das Alter des Gründers und der Erfolg der geförderten Gründung in unserer Studie negativ zusammen. Unsere Erklärung dafür ist, dass ältere Menschen im Durchschnitt höhere Lebenshaltungskosten haben und der aus der Existenzgründung erwirtschaftete Gewinn daher auch höher sein muss. Förderprogramme für Gründungen durch ältere Menschen sollten diesen Aspekt berücksichtigen. Zweitens, Gründer, die vor der Gründung sehr lange arbeitslos waren, waren im Durchschnitt weniger erfolgreich als andere Gründer. Hier stellt sich aus Perspektive der Gründungspolitik die Frage, ob nicht stärkere Anreize gesetzt werden sollten, das Gründungsvorhaben zügig umzusetzen, um so die Erfolgschancen der Gründung zu erhöhen. Vor diesem Hintergrund erscheint die zuletzt diskutierte und vereinbarte Verlängerung des Arbeitslosengeldes für Ältere nicht sinnvoll. Gleiches gilt auch für die bereits bestehende und weit verbreitete Praxis, den Arbeitslosengeld-I-Anspruch durch ein vorgeschaltetes Transferkurzarbeitergeld in gleicher Höhe um ein Jahr zu verlängern.

Aufgrund der Limitationen unseres Datensatzes und der aufgeworfenen Fragen ergibt sich weiterer Forschungsbedarf. Interessante, zu untersuchende Fragen wären z.B.: Wie erfolgreich sind die geförderten Existenzgründungen über einen längeren Zeitraum hin betrachtet? Was passiert mit denjenigen Existenzgründungen, deren Gewinn (noch) nicht zur Bestreitung des Lebensunterhalts ausreicht? In methodischer Hinsicht wäre ein repräsentativ erhobener Paneldatensatz wünschenswert. So könnte besser auf Selektionseffekte und unbeobachtete Heterogenität ${ }^{9}$ kontrolliert werden. 


\section{Anmerkungen}

1 Das Instrument Überbrückungsgeld wurde am 1.1.1986, der Existenzgründungszuschuss wurde am 1.1.2003 eingeführt.

2 Die Begriffe Necessity- bzw. Opportunity-Gründer wurden durch den Global Entrepreneurship Monitor (GEM) geprägt. Necessity-Gründer sind solche Gründer, die aus Mangel an Alternativen ein Unternehmen gründen, während Opportunity-Gründer mit der Unternehmensgründung eine unternehmerische Idee umsetzen wollen (vgl. Reynolds et. al., 2001, 2005, Galais, 1998; vgl. zur Schwierigkeit der Abgrenzung Block/Wagner, 2006).

${ }^{3}$ In der politischen Diskussion wird in diesem Zusammenhang von tragfähigen Gründungen gesprochen.

4 Die Bewertung durch Arbeitsmarktexperten ist positiv. Aufgezeigt wird, dass das Förderinstrument „Überbrückungsgeld“ das Arbeitslosigkeitsrisiko der Empfänger (nach Ablauf der Förderung) deutlich verringert und $\mathrm{zu}$ einer positiven Einkommensentwicklung beiträgt. Auch aus Effizienzsicht war das Instrument ein Erfolg. So wurde im Vergleich zu der Situation, dass die Empfänger weiterhin Arbeitslosenunterstützung bezogen hätten, Geld eingespart (vgl. Caliendo/Steiner, 2007; Caliendo et al., 2006).

${ }^{5}$ Eine Förderung (z.B. durch den Gründungszuschuss ist auch nach einer Eigenkündigung möglich. Wenn selbst gekündigt oder einem Aufhebungsvertrag zugestimmt wird, verhängt die Arbeitsagentur eine Sperrzeit von in der Regel drei Monaten. Diese Sperrzeit kann vermeiden werden, wenn ein wichtiger Grund nachgewiesen wird (z.B gesundheitliche Gründe oder der Umzug zum Ehepartner). Nach Ende der Sperrzeit besteht in der Regel ein Anspruch auf Förderung (vgl. § 144 SGB III sowie Zeile 57.31 in der Durchführungsanweisung zum Gründungszuschuss-Paragraphen).

6 Diese 33.695 Personen setzen sich zusammen aus 8.857 durch unseren Kooperationspartners gruendungszuschuss.de angeschriebene Kunden sowie 24.838 Abonnenten seines Newsletters.

7 Zur Schätzung verwenden wir den heckprob-Befehl, der in Stata 9 implementiert ist. 
${ }^{8}$ Klandt et al. (1998) untersuchen ebenfalls geförderte Gründungen und finden ähnlich wie wir keinen Erfolgsunterschied zwischen Einzel- und Teamgründungen.

9 Selektionseffekte verzerren die Ergebnisse und sind in der Auswahl der Stichprobe begründet. Unbeobachtete Heterogenität liegt vor, wenn es außer den betrachteten unabhängigen Variablen noch weitere Variablen gibt, die ebenfalls einen Einfluß auf die abhängige Variable haben. 


\section{Literatur}

Albach, H. (2005), Unternehmensethik: ein subjektiver Überblick, in: Zeitschrift für Betriebswirtschaft Special Issue 5, S. 3-36.

Amit, R., Muller, E., Cockburn, I. (1995), Opportunity Costs and Entrepreneurial Activity, Journal of Business Venturing 10(2), 95-106.

Becker, G. (1964), Human Capital, Chicago, University of Chicago Press.

Bendick, M., Egan, M. L. (1987), Transfer Payment Diversion for Small Business Development: British and French Experience, Industrial and Labour Relations Review 40, 428-542.

Bergmann, H. and R. Sternberg. (2007), The Changing Face of Entrepreneurship in Germany, Small Business Economics 28, 205-221.

Block, J., Sandner, P. (2007), Necessity and Opportunity Entrepreneurs and their Duration in Self-employment: Evidence from German Micro Data, Journal of Industry, Competition and Trade, forthcoming in 2007.

Block, J., Wagner, M. (2006), Opportunity Recognition and Exploitation by Necessity and Opportunity Entrepreneurs: Empirical Evidence from Earnings Equations, SSRN Working Paper, http://ssrn.com/abstract=899968.

Brüderl, J., Preisendörfer, P. and R. Ziegler. (1992), Survival Chances of Newly Founded Founded Business Organizations, American Sociological Review 57, $227-242$.

Brüderl, J., Preisendörfer, P. (1998), Network Support and the Success of Newly Founded Business, in: Small Business Economics 10, 213-225.

Brüderl, J., Preisendörfer, P., Ziegler, R. (1998), Der Erfolg neugegründeter Betriebe - Eine empirische Studie zu den Chancen und Risiken von Unternehmensgründungen, 2. Auflage, Berlin.

Bundesministerium der Justiz (2007), § 57 SGB III „Gründungszuschuss“, http://bundesrecht.juris.de/sgb_3/_57.html.

Bundesagentur für Arbeit (2007), http://www.arbeitsagentur.de, Zugriff am 5. Juli 2007. 
Caliendo, M., Kritikos, A. S., Wießner, F. (2006), Existenzgründungsförderung in Deutschland - Zwischenergebnisse aus der Hartz-Evaluation, in: Zeitschrift für Arbeitsmarkforschung 39, 503-529.

Caliendo, M., Steiner, V. (2007), Ich-AG und Überbrückungsgeld - Neue Ergebnisse bestätigen Erfolg, in: DIW Wochenbericht 3, 25-32.

Carr, D. (1996), Two Paths to Self-Employment?: Women's and Men's Self-Employment in the United States, 1980, Work and Occupations 23, 26-53.

Davidsson, P. and Honig, B. (2003). “The Role of Social and Human Capital among Nascent Entrepreneurs.” Journal of Business Venturing 18, 301-331.

Duchesneau, D. A., Gartner, W. B. (1990), A Profile of New Venture Success and Failure in An Emerging Industry, Journal of Business Venturing 5(5), 297-312.

Devine, T. J., Kiefer, N. M. (1993), The Empirical Status of Job Search Theory, in: Labour Economics 1, 3-24.

Ehrmann, T. (2007), Der ehrbare Frachisegeber: Ein empirischer Beitrag, in: ZfB Special Issue 1.

Evans, D. S., Leighton, L. S. (1989), Some Empirical Aspects of Entrepreneurship, in: American Economic Review 79, 519-535.

Fallgatter, M. (2002), Theorie des Entrepreneurship - Perspektiven zur Erforschung der Entstehung und Entwicklung junger Unternehmungen, Wiesbaden.

Galais, N. (1998), Motive und Beweggründe für die Selbstständigkeit und ihre Bedeutung für den Erfolg, in: Frese, M. (Hrsg.): Erfolgreiche Unternehmensgründer - Psychologische Analysen und praktische Anleitungen für Unternehmer in Ost- und Westdeutschland, Göttingen, S. 83-98.

Heckman, J. (1976), The Common Structure of Statistical Models of Truncation, Sample Selection and Limited Dependent Variables and a Simple Estimator for Such Models, in: Annals of Economic and Social Measurement 5(4), S. 475-492. 
Hinz, T., Jungbauer-Gans, M. (1999), Starting a Business after Unemployment: Characteristics and Chances of Success (Empirical Evidence from Regional German Labour Market), Entrepreneurship and Regional Development 11, 317-333.

Jack, S., Anderson, A. (2002), The Effects of Embeddedness on the Entrepreneurial Process, in: Journal of Business Venturing 17: 467-488.

Jacobsen, L. K. (2006), Erfolgsfaktoren bei der Unternehmensgründung, Deutscher Universitätsverlag, Wiesbaden.

Jovanovic, B. (1982), Selection and the Evolution of Industry, in: Econometrica 50: 649-670.

Jungbauer-Gans, M., Preisendörfer, P. (1991), Verbessern eine gründliche Vorbereitung und sorgfältige Planung die Erfolgschancen neugegründeter Betriebe, in: Zeitschrift für betriebswirtschaftliche Forschung 43, 987-996.

Kahneman, D., Knetsch, J. L., Thaler, R. H. (1991), Anomalies: The Endowment Effect, Loss Aversion, and Status Quo Bias, in: The Journal of Economic Perspectives 5, 193-206.

Klandt, H., Kirchhoff-Kestel, S., Struck, J. (1998), Zur Wirkung der Existenzgründererförderung auf junge Unternehmen - eine vergleichende Analyse geförderter und nicht-geförderter Unternehmen, FGF Entrepreneurship Research Monographien, Köln.

Lauxen-Ulbricht, M., Leicht, R. A. (2002), Entwicklung und Tätigkeitsprofil selbständiger Frauen in Deutschland, Institut für Mittelstandsforschung, Universität Mannheim, 1-78.

Lauxen-Ulbrich, M., Leicht, R. (2005): Wie Frauen gründen und was sie unternehmen: Nationaler Report Deutschland-Teilprojekt, Institut für Mittelstandsforschung, Universität Mannheim.

Lehnert, N. (2004), KfW-Gründungsmonitor 2004 - Zahl der Vollerwerbsgründungen stabil Gründungen aus der Arbeitslosigkeit gewinnen an Bedeutung, Kreditanstalt für Wiederaufbau, Frankfurt.

Lutz, A. (2007), Gründungszuschuss und Einstiegsgeld - Erfolgreich selbständig mit Geld vom Staat, Wien. 
Mathewson, F., Winter, R. A. (1985), The Economics of Franchise Contracts, in: Journal of Law and Economics 28, 503-526.

Meager, N. (1996), From Unemployment to Self-Employment: Labour Market Policies for Business Start-up, in: Schmidt, G., O’Reilly, J., Schömann, K. (Hrsg.): International Handbook of Labour Market Policy and Evaluation, Cheltenham, 489-519.

Mellewigt, T., Witt, P. (2002), Die Bedeutung des Vorgründungsprozesses für die Evolution von Unternehmen, in: Zeitschrift für Betriebswirtschaft 72, 81-110.

Mincer, J. (1974), Schooling, Experience and Earnings, New York.

Nelson, R. R., Winter, S. G. (1982), An Evolutionary Theory of Economic Change, Cambridge, Mass.

Niefert, M., Tchouvakhina, M. (2006), Gründungen aus der Arbeitslosigkeit-Besondere Merkmale und Unterschiede zu anderen Gründungen, in: Mittelstands und Strukturpolitik 25, 109-123.

Parker, S. C. (2004), The Economics of Self-Employment and Entrepreneurship, New York.

Pfeiffer, F., Reize, F. (2000), Business Start-ups by the Unemployed - An Econometric Analysis based on Firm Data, in: Labour Economics, 629-663.

Picot, A., Laub, U. D., Schneider, Schneider, D. (1989), Innovative Unternehmensgründungen. Eine ökonomisch-empirische Analyse, Berlin et al.

Praag, C. M. van (2003), Business Survival and Success of Young Small Business Owners, in: Small Business Economics 21, 1-17.

Reize, F. (2000), Leaving Unemployment for Self-Employment. A Discrete Duration Analysis of Determinants and Stability of Self-Employed among Former Unemployed, Discussion Paper 00-06, ZEW.

Reynolds, P. D., Bosma, N., Autio, E., Hunt, S., Bono, N., Servais, I., Lopez-Garcia, P., Chin, N. (2005), Global Entrepreneurship Monitor: Data Collection Design and Implementation 1998-2003, in: Small Business Economics 24, 205-231. 
Reynolds, P. D., Camp, S. M., Bygrave, W. D., Autio, E., Hay, M, Global Entrepreneurship Monitor 2001 Executive Report, Babson College, London Business School.

Romanelli, E., Schoonhoven, C. B. (2001), The Local Origins of New Firms, in: The Entrepreneurship Dynamic Origins of Entrepreneurship and the Evolution of Industries, Stanford, Stanford University Press, 40-67.

Ruspini, E. (2002), Introduction to Longitudinal Research, London.

Schmidt, A. G. (2002): Indikatoren für Erfolg und Überlebenschancen junger Unternehmen, in: Zeitschrift für Betriebswirtschaft, Ergänzungsheft 5, 21-53.

Schwarz, E. J., Ehrmann, T., Breitenecker, R. J. (2005), Erfolgsdeterminanten junger Unternehmen in Österreich: eine empirische Untersuchung zum Beschäftigungswachstum, in: Zeitschrift für Betriebswirtschaft 11, 1077-1098.

Shane, S. (2000), Prior Knowledge and the Discovery of Entrepreneurial Opportunities, in: Organization Science 11, 448-469.

Shane, S. (2003), A General Theory of Entrepreneurship - The Individual-Opportunity Nexus, Cheltenham.

Sternberg, R., Brixy, U., Hundt, C. (2007), Global Entrepreneurship Monitor (GEM) - Länderbericht Deutschland 2006, Leibnitz Universität Hannover und Institut für Arbeitsmarktund Berufsforschung (IAB).

Szyperski, N. (1999), Meinungen zum Thema: Existenzgründungen, in: Betriebswirtschaftliche Forschung und Praxis 51 (3), S. 322-332.

Szyperski, N., Nathusius, K. (1999), Probleme der Unternehmensgründung: eine betriebswirtschaftliche Analyse unternehmerischer Startbedingungen, 2. Auflage, München.

Wießner, F. (2000), Erfolgsfaktoren von Existenzgründungen aus der Arbeitslosigkeit - Eine multivariate Betrachtung vormals arbeitsloser Existenzgründer, die vom Arbeitsamt mit dem Überbrückungsgeld ( $\$ 57$ SGB III) gefördert wurden, in: Mitteilungen aus der Arbeitsmarktund Berufsforschung 33, 518-532.

Wooldridge, J. M. (2002): Introductory Econometrics: A Modern Approach, 2. Ausgabe. 
Tab. 1: Beschreibung der einzelnen Förderinstrumente

\begin{tabular}{|c|c|c|c|c|}
\hline Charakteristika & $\begin{array}{c}\text { Überbrückungs- } \\
\text { geld }\end{array}$ & $\begin{array}{l}\text { Existenzgrün- } \\
\text { dungszuschuss } \\
\text { ("Ich-AG") }\end{array}$ & Einstiegsgeld & $\begin{array}{l}\text { Gründungs- } \\
\text { zuschuss }\end{array}$ \\
\hline Rechtsgrundlage & $\begin{array}{l}\S 57 \text { (SGB III) } \\
\text { in Fassung } \\
\text { bis } 31.7 .2006\end{array}$ & $\S 4211$ (SGB III) & $\S 29$ (SGB II) & $\begin{array}{l}\S 57 \text { (SGB III) } \\
\text { in Fassung } \\
\text { ab } 1.8 .2006\end{array}$ \\
\hline Anspruchsgrundlage & $\begin{array}{l}\text { ALG1-Anspruch, } \\
\text { bis 31.12.2004 auch } \\
\text { Arbeitslosenhilfe }\end{array}$ & $\begin{array}{l}\text { ALG1-Anspruch, } \\
\text { bis 31.12.2004 auch } \\
\text { Arbeitslosenhilfe }\end{array}$ & ALG2-Anspruch & $\begin{array}{l}\text { Mind. } 90 \text { Tage } \\
\text { ALG1-Restanspruch }\end{array}$ \\
\hline $\begin{array}{l}\text { Notwendigkeit von } \\
\text { Businessplan und fach- } \\
\text { kundige Stellungnahme }\end{array}$ & $\mathrm{Ja}$ & $\begin{array}{l}\text { Ja, seit Herbst 2004, } \\
\text { zuvor nicht }\end{array}$ & $\begin{array}{l}\text { In der Regel ja, aber } \\
\text { Ermessensentschei- } \\
\text { dung }\end{array}$ & $\begin{array}{l}\text { Ja, zusätzlich "Dar- } \\
\text { legung der persönli- } \\
\text { chen Eignung" }\end{array}$ \\
\hline Gültig seit/bis & $\begin{array}{l}1.1 .1986 \text { bis } \\
1.11 .2006\end{array}$ & $\begin{array}{l}\text { 1.1.2003 bis } \\
30.6 .2006\end{array}$ & Seit 1.1.2005 & Seit 1.8.2006 \\
\hline Rechtsanspruch & Ja, seit 1.1.2004 & $\mathrm{Ja}$ & $\begin{array}{l}\text { Nein, Kann- } \\
\text { Leistung }\end{array}$ & $\begin{array}{l}\text { Ja, für erste } 9 \text { Mona- } \\
\text { te und von } 10 . \text { bis } \\
15 \text {. Monat Kann- } \\
\text { Leistung }\end{array}$ \\
\hline $\begin{array}{l}\text { Höhe der Förderung pro } \\
\text { Monat/Dauer }\end{array}$ & $\begin{array}{l}\text { In Höhe des ALG1- } \\
\text { Anspruchs plus ca. } \\
70 \% \text { Zuschlag für } \\
6 \text { Monate }\end{array}$ & $\begin{array}{l}\text { Gestaffelt: } \\
600 \text { Euro (1. Jahr), } \\
360 \text { Euro (2. Jahr), } \\
\text { 240 Euro (3. Jahr), } \\
\text { insgesamt maximal } \\
\text { 14.400 Euro }\end{array}$ & $\begin{array}{l}\text { Zusätzlich zum } \\
\text { ALG2: } 50 \% \text { der } \\
\text { Regelleistung ( } 345 \\
\text { Euro) zzgl. } 10 \% \text { pro } \\
\text { weiteres Mitglied } \\
\text { der Bedarfsgemein- } \\
\text { schaft für in der } \\
\text { Regel } 6 \text { bis } 12 \text { Mo- } \\
\text { nate }\end{array}$ & $\begin{array}{l}\text { In Höhe des ALG1- } \\
\text { Anspruchs plus } 300 \\
\text { Euro (erste } 9 \text { Mona- } \\
\text { te), anschließend nur } \\
\text { 300 Euro (6 Mona- } \\
\text { te) }\end{array}$ \\
\hline $\begin{array}{l}\text { Verrechnung mit } \\
\text { ALG1-Restanspruch }\end{array}$ & Nein & Nein & $\begin{array}{l}\text { Entfällt, da auf } \\
\text { Grundlage ALG2 }\end{array}$ & $\mathrm{Ja}$ \\
\hline $\begin{array}{l}\text { Obergrenze für selb- } \\
\text { ständiges Einkommen }\end{array}$ & $\begin{array}{l}\text { Ex post nein, ex ante } \\
\text { (Businessplan) muss } \\
\text { Förderbedarf er- } \\
\text { kennbar sein }\end{array}$ & $\begin{array}{l}\text { Bei }>25.000 \text { Euro } \\
\text { Gewinn p.a. entfällt } \\
\text { Förderung in Folge- } \\
\text { jahren }\end{array}$ & $\begin{array}{l}\text { Weitgehende Ver- } \\
\text { rechnung des Ge- } \\
\text { winns mit ALG2- } \\
\text { Anspruch }\end{array}$ & $\begin{array}{l}\text { Ex post nein, ex ante } \\
\text { (Businessplan) muss } \\
\text { Förderbedarf er- } \\
\text { kennbar sein }\end{array}$ \\
\hline $\begin{array}{l}\text { Gesetzliche Renten-, } \\
\text { Kranken- und Pflege- } \\
\text { versicherung }\end{array}$ & Freiwillig möglich & $\begin{array}{l}\text { Pflichtversichert in } \\
\text { gesetzlicher Renten- } \\
\text { versicherung, ermä- } \\
\text { ßigter Mindestbei- } \\
\text { trag in der gesetzli- } \\
\text { chen Kranken- und } \\
\text { Pflegeversicherung }\end{array}$ & $\begin{array}{l}\text { Im Rahmen des } \\
\text { ALG2-Bezugs } \\
\text { sozialversichert }\end{array}$ & $\begin{array}{l}\text { Freiwillig möglich, } \\
\text { ermäßigter Mindest- } \\
\text { beitrag in der ge- } \\
\text { setzlichen Kranken- } \\
\text { und Pflegeversiche- } \\
\text { rung }\end{array}$ \\
\hline \multicolumn{5}{|l|}{$\begin{array}{l}\text { Anzahl geförderter } \\
\text { Gründungen }{ }^{1}\end{array}$} \\
\hline-2000 & 92.596 & 0 & 0 & 0 \\
\hline-2001 & 95.926 & 0 & 0 & 0 \\
\hline-2002 & 124.885 & 0 & 0 & 0 \\
\hline-2003 & 158.696 & 95.198 & 0 & 0 \\
\hline-2004 & 183.179 & 168.176 & 0 & 0 \\
\hline-2005 & 156.888 & 91.020 & 17.149 & 0 \\
\hline-2006 & 108.266 & 42.812 & 32.571 & 33.569 \\
\hline-2007 & 0 & 0 & 23.926 & 92.211 \\
\hline
\end{tabular}

ALG1=Arbeitslosengeld I, ALG2=Arbeitslosengeld II.

ALG1=Arbeitslosengeld I, ALG2=Arbeitslosengeld II.
vorläufige Zahlen von Januar 2007 bis September 2007, Quelle: Bundesagentur für Arbeit (2007); Lutz (2007). 
Tab. 2: Beschreibung der in der Studie verwendeten Variablen

\section{Kurzbezeichnung(en) \\ Gründungsvorbereitung \\ BUSINESSPLANUMFANG \\ BERATUNGSUNTERSTÜTZUNG

\author{
Gründungserfolg \\ ERFOLG \\ GRÜNDUNGSZUFRIEDENHEIT \\ GRÜNDUNG WIEDERHOLEN
} \\ Gründungsmotivation \\ NECESSITY \\ ARBEITSLOSIGKEIT

Gründungscharakteristika
FREMDKAPITAL
FRANCHISE
TEAM

Demographische Merkmale

ALTER

GESCHLECHT

DEUTSCH

\section{Humankapital \\ BRANCHENERFAHRUNG \\ GRÜNDUNGSERFAHRUNG \\ FÜHRUNGSERFAHRUNG}

REALSCHULE, ABITUR, BERUFSAUSBILDUNG, MEISTERPRÜFUNG, STUDIUM, PROMOTION, KEINE ANGABE

Förderungsinstrumente

ICH-AG, ÜBERBRÜCKUNGSGELD, GRÜNDUNGSZUSCHUSS, EINSTIEGSGELD

\section{Region}

PLZ0-PLZ9

\section{Branchenvariablen}

BRANCHE1-BRANCHE12

\section{Ausprägungen der Variable(n)}

Umfang des Businessplans ohne Inhaltsverzeichnis, Zahlenteil und Anhang (ordinale Skalierung, 1=keinen Businessplan, 2=1-2 Seiten, 3=3-5 Seiten, 4=6-10 Seiten, 5=11-15 Seiten, $6=16-20$ Seiten, $7=$ mehr als 20 Seiten).

Variable $=1$, wenn beratende Unterstützung bei der Erstellung des Businessplans erfolgte.

Variable $=1$, wenn der Gewinn zum Lebensunterhalt ausreicht.

Zufriedenheit des Gründers mit seiner Gründung (1=sehr unzufrieden; 5=sehr zufrieden). Variable=1, wenn der Gründer seine Entscheidung zur Selbständigkeit retrospektiv betrachtet nochmals so treffen würde und wieder gründen würde.

Variable=1, wenn der Gründer sein letztes Arbeitsverhältnis vor dem Eintritt in die Selbständigkeit unfreiwillig verlassen hat.

Anzahl der Monate, die der Gründer vor dem Eintritt in die Selbständigkeit arbeitslos war.

Variable $=1$, wenn der Gründer Fremdkapital aufgenommen hat.

Variable $=1$, wenn es sich um eine Franchisegründung handelt.

Variable $=1$, wenn das Unternehmen von mindestens zwei Personen gegründet worden ist.

Alter des Gründers in Jahren

Variable $=1$, wenn der Gründer männlich ist.

Variable $=1$, wenn es sich um einen Gründer mit deutscher Nationalität handelt.

Variable $=1$, wenn der Gründer in der Branche der Gründung bereits Berufserfahrung hat. Variable $=1$, wenn der Gründer vor der Gründung bereits einmal selbständig war. Variable=1, wenn der Gründer vor der Gründung bereits Führungserfahrung gesammelt hat.

7 Dummyvariablen, die den höchsten erreichten Bildungsabschluss des Gründers angeben: Mindestens Realschule, Abitur, abgeschlossene Berufsausbildung, Meisterprüfung, abgeschlossenes Hochschulstudium (FH oder Uni), Promotion, keine Angabe.

4 Dummyvariablen für die Art des in Anspruch genommenen Förderinstrumentes: Existenzgründungszuschuss (Ich-AG), Überbrückungsgeld, Gründungszuschuss oder Einstiegsgeld.

10 Dummyvariablen, die angeben, in welchem Postleitzahlengebiet die Gründung erfolgte: PLZ-Gebiet 0-9.

12 Dummyvariablen, die angeben, in welcher Branche die Gründung erfolgte: Baugewerbe (inkl. Ingenieurwesen und Architekt), Beratung/Recht/Training, kaufmännische Dienstleistung, Gastronomie, Gesundheit/Kosmetik, Handel/Einzelhandel, Handwerk, IT/Software/Internet, Marketing/Event-Management/Kunst (inkl. Medien), Service (z.B. Haushalt, Reinigung, Transport), Vertrieb (z.B. Finanzvertrieb), Sonstiges.

4 Dummyvariablen für das Jahr der Gründung: 2003-2006. 
Tab. 3: Deskriptive Statistiken

\begin{tabular}{|c|c|c|c|c|}
\hline Variable & $\begin{array}{c}\text { Mittel- } \\
\text { Wert/ } \\
\text { Anteil }\end{array}$ & $\begin{array}{l}\text { Std.- } \\
\text { Abw. }\end{array}$ & Min. & Max. \\
\hline ERFOLG & $65 \%$ & & 0 & 1 \\
\hline GRUENDUNGSZUFRIENDENHEIT & 3,982 & 0,843 & 1 & 5 \\
\hline GRÜNDUNG WIEDERHOLEN & $97 \%$ & & 0 & 1 \\
\hline \multicolumn{5}{|l|}{ Gründungsmotivation } \\
\hline NECESSITY & $68 \%$ & & 0 & 1 \\
\hline ARBEITSLOSIGKEIT & 4,702 & 5,540 & 0 & 21 \\
\hline \multicolumn{5}{|l|}{ Gründungsvorbereitung } \\
\hline BUSINESSPLANUMFANG & 4,565 & 1,515 & 1 & 7 \\
\hline BERATUNGSUNTERSTÜTZUNG & $76 \%$ & & 0 & 1 \\
\hline \multicolumn{5}{|l|}{ Gründungscharakteristika } \\
\hline FREMDKAPITAL & $30 \%$ & & 0 & 1 \\
\hline FRANCHISE & $2 \%$ & & 0 & 1 \\
\hline TEAM & $15 \%$ & & 0 & 1 \\
\hline \multicolumn{5}{|l|}{ Demographische Merkmale } \\
\hline ALTER & 40,959 & 7,963 & 16 & 62 \\
\hline GESCHLECHT & $69 \%$ & & 0 & 1 \\
\hline DEUTSCH & $95 \%$ & & 0 & 1 \\
\hline \multicolumn{5}{|l|}{ Humankapital } \\
\hline BRANCHENERFAHRUNG & $76 \%$ & & 0 & 1 \\
\hline GRÜNDUNGSERFAHRUNG & $33 \%$ & & 0 & 1 \\
\hline FÜHRUNGSERFAHRUNG & $65 \%$ & & 0 & 1 \\
\hline BILDUNG: REALSCHULE & $7 \%$ & & 0 & 1 \\
\hline BILDUNG: ABITUR & $10 \%$ & & 0 & 1 \\
\hline BILDUNG: BERUFSAUSBILDUNG & $21 \%$ & & 0 & 1 \\
\hline BILDUNG: MEISTERPRÜFUNG & $4 \%$ & & 0 & 1 \\
\hline BILDUNG: STUDIUM & $47 \%$ & & 0 & 1 \\
\hline BILDUNG: PROMOTION & $4 \%$ & & 0 & 1 \\
\hline BILDUNG: KEINE ANGABE & $5 \%$ & & 0 & 1 \\
\hline \multicolumn{5}{|l|}{ Förderungsinstrumente } \\
\hline $\mathrm{ICH}-\mathrm{AG}$ & $8 \%$ & & 0 & 1 \\
\hline ÜBERBRÜCKUNGSGELD & $71 \%$ & & 0 & 1 \\
\hline GRÜNDUNGSZUSCHUSS & $19 \%$ & & 0 & 1 \\
\hline EINSTIEGSGELD & $2 \%$ & & 0 & 1 \\
\hline \multicolumn{5}{|l|}{ Gründungsjahr } \\
\hline JAHR2003 & $4 \%$ & & 0 & 1 \\
\hline JAHR2004 & $14 \%$ & & 0 & 1 \\
\hline JAHR2005 & $29 \%$ & & 0 & 1 \\
\hline JAHR2006 & $53 \%$ & & 0 & 1 \\
\hline \multicolumn{5}{|l|}{ Branchenvariablen } \\
\hline BRANCHE1: Baugewerbe & $7 \%$ & & 0 & 1 \\
\hline BRANCHE2: Beratung, Recht, Training & $15 \%$ & & 0 & 1 \\
\hline BRANCHE3: kaufm. Dienstleistung & $6 \%$ & & 0 & 1 \\
\hline BRANCHE4: Gastronomie & $1 \%$ & & 0 & 1 \\
\hline BRANCHE5: Gesundheit, Kosmetik & $4 \%$ & & 0 & 1 \\
\hline BRANCHE6: Handel, Einzelhandel & $8 \%$ & & 0 & 1 \\
\hline BRANCHE7: Handwerk & $3 \%$ & & 0 & 1 \\
\hline BRANCHE8: IT, Software, Internet & $17 \%$ & & 0 & 1 \\
\hline BRANCHE9: Marketing, Kultur, Events & $16 \%$ & & 0 & 1 \\
\hline BRANCHE10: Service & $1 \%$ & & 0 & 1 \\
\hline BRANCHE11: Vertrieb & $5 \%$ & & 0 & 1 \\
\hline BRANCHE12: Sonstiges & $17 \%$ & & 0 & 1 \\
\hline
\end{tabular}


Tab. 4: Erfolgreiche und nicht erfolgreiche Existenzgründer im Vergleich

\begin{tabular}{|c|c|c|c|}
\hline Variable & $\begin{array}{c}\text { Mittelwert/Anteil } \\
\text { bei erfolgreichen } \\
\text { Existenzgründern } \\
(\mathbf{N}=719)\end{array}$ & $\begin{array}{l}\text { Mittelwert/Anteil } \\
\text { bei nicht } \\
\text { erfolgreichen }^{1} \\
\text { Existenzgründern } \\
(\mathbf{N}=\mathbf{3 8 7})\end{array}$ & p-Wert \\
\hline GRÜNDUNGSZUFRIEDENHEIT & 3,822 & 2,550 & $0,000^{2}$ \\
\hline GRÜNDUNG WIEDERHOLEN & $99 \%$ & $92 \%$ & 0,000 \\
\hline \multicolumn{4}{|l|}{ Gründungsmotivation } \\
\hline NECESSITY & $63 \%$ & $77 \%$ & 0,000 \\
\hline ARBEITSLOSIGKEIT & 3,573 & 6,798 & $0,000^{2}$ \\
\hline \multicolumn{4}{|l|}{ Gründungscharakteristika } \\
\hline FREMDKAPITAL & $30 \%$ & $30 \%$ & 0,980 \\
\hline FRANCHISE & $2 \%$ & $4 \%$ & 0,023 \\
\hline TEAM & $16 \%$ & $13 \%$ & 0,154 \\
\hline \multicolumn{4}{|l|}{ Demographische Merkmale } \\
\hline ALTER & 40,065 & 42,620 & $0,000^{2}$ \\
\hline GESCHLECHT & $75 \%$ & $57 \%$ & 0,000 \\
\hline DEUTSCH & $96 \%$ & $94 \%$ & 0,368 \\
\hline \multicolumn{4}{|l|}{ Humankapital } \\
\hline BRANCHENERFAHRUNG & $81 \%$ & $65 \%$ & 0,000 \\
\hline GRÜNDUNGSERFAHRUNG & $33 \%$ & $33 \%$ & 0,938 \\
\hline FÜHRUNGSERFAHRUNG & $67 \%$ & $62 \%$ & 0,104 \\
\hline BILDUNG: REALSCHULE & $7 \%$ & $7 \%$ & 0,660 \\
\hline BILDUNG: ABITUR & $9 \%$ & $11 \%$ & 0,118 \\
\hline BILDUNG: BERUFSAUSBILDUNG & $20 \%$ & $24 \%$ & 0,089 \\
\hline BILDUNG: MEISTERPRÜFUNG & $5 \%$ & $3 \%$ & 0,328 \\
\hline BILDUNG: STUDIUM & $49 \%$ & $43 \%$ & 0,049 \\
\hline BILDUNG: PROMOTION & $5 \%$ & $3 \%$ & 0,108 \\
\hline \multicolumn{4}{|l|}{ Förderungsinstrumente } \\
\hline $\mathrm{ICH}-\mathrm{AG}$ & $7 \%$ & $11 \%$ & 0,018 \\
\hline ÜBERBRÜCKUNGSGELD & $73 \%$ & $66 \%$ & 0,010 \\
\hline GRÜNDUNGSZUSCHUSS & $19 \%$ & $20 \%$ & 0,548 \\
\hline EINSTIEGSGELD & $1 \%$ & $3 \%$ & 0,020 \\
\hline \multicolumn{4}{|l|}{ Branchenvariablen } \\
\hline BRANCHE1: Baugewerbe & $9 \%$ & $3 \%$ & 0,001 \\
\hline BRANCHE2: Beratung, Recht, Training & $16 \%$ & $13 \%$ & 0,109 \\
\hline BRANCHE3: kaufm. Dienstleistung & $7 \%$ & $6 \%$ & 0,635 \\
\hline BRANCHE4: Gastronomie & $1 \%$ & $2 \%$ & 0,096 \\
\hline BRANCHE5: Gesundheit, Kosmetik & $4 \%$ & $6 \%$ & 0,107 \\
\hline BRANCHE6: Handel, Einzelhandel & $5 \%$ & $11 \%$ & 0,000 \\
\hline BRANCHE7: Handwerk & $3 \%$ & $3 \%$ & 0,954 \\
\hline BRANCHE8: IT, Software, Internet & $18 \%$ & $15 \%$ & 0,113 \\
\hline BRANCHE9: Marketing, Kultur, Events & $15 \%$ & $17 \%$ & 0,468 \\
\hline BRANCHE10: Service & $1 \%$ & $2 \%$ & 0,682 \\
\hline BRANCHE11: Vertrieb & $4 \%$ & $7 \%$ & 0,025 \\
\hline BRANCHE12: Sonstiges & $17 \%$ & $17 \%$ & 0,844 \\
\hline
\end{tabular}

$\mathrm{N}=1.106$

${ }^{1}$ Ein Unternehmer gilt als erfolgreich, wenn der Gewinn zur Bestreitung des Lebensunterhalts ausreicht.

2 Diese gekennzeichneten p-Werte basieren auf t-Tests, wohingegen nicht gekennzeichnete p-Werte aus Chi²-Anteilswert-Tests stammen.

Anmerkung: Ein p-Wert kleiner als 0,05 bedeutet, dass die Null-Hypothese gleicher Mittelwerte auf einem Fehlerniveau $\leq 5 \%$ zurückgewiesen werden kann. 
Tab. 5: Korrelationstabelle verwendeter Variablen ${ }^{1}$

\begin{tabular}{|c|c|c|c|c|c|c|c|c|c|c|c|c|}
\hline Variablen & (1) & $(2)$ & (3) & (4) & (5) & (6) & (7) & (8) & (9) & (10) & (11) & VIF $^{2}$ \\
\hline \multicolumn{13}{|l|}{ (1) ERFOLG } \\
\hline \multicolumn{13}{|l|}{ Gründungsmotivation } \\
\hline (2) NECESSITY & $-0,14^{*}$ & & & & & & & & & & & 1,31 \\
\hline (3) ARBEITSLOSIGKEIT & $-0,28^{*}$ & $0,37^{*}$ & & & & & & & & & & 1,40 \\
\hline \multicolumn{13}{|l|}{ Gründungscharakteristika } \\
\hline (4) FREMDKAPITAL & $-0,00$ & $-0,04$ & $-0,01$ & & & & & & & & & 1,13 \\
\hline (5) FRANCHISE & $-0,07^{*}$ & $-0,03$ & $-0,05$ & $0,10^{*}$ & & & & & & & & 1,05 \\
\hline (6) TEAM & 0,04 & $-0,12^{*}$ & $-0,10^{*}$ & $0,12^{*}$ & $-0,02$ & & & & & & & 1,11 \\
\hline \multicolumn{13}{|l|}{ Demographische Merkmale } \\
\hline (7) ALTER & $-0,15^{*}$ & $0,25^{*}$ & $0,31 *$ & $-0,01^{*}$ & $-0,05$ & $-0,10^{*}$ & & & & & & 1,28 \\
\hline (8) GESCHLECHT & $0,18^{*}$ & 0,01 & $-0,07^{*}$ & 0,01 & 0,02 & $0,10^{*}$ & $0,06^{*}$ & & & & & 1,20 \\
\hline (9) DEUTSCH & 0,03 & 0,02 & $-0,04$ & $-0,04$ & $-0,02$ & 0,03 & 0,02 & 0,05 & & & & 1,06 \\
\hline \multicolumn{13}{|l|}{ Humankapital } \\
\hline (10) BRANCHENERFAHRUNG & $0,18^{*}$ & $-0,01$ & $-0,13^{*}$ & $-0,05$ & $-0,09^{*}$ & $0,06^{*}$ & $-0,01$ & $0,12^{*}$ & 0,05 & & & 1,17 \\
\hline (11) GRÜNDUNGSERFAHRUNG & $-0,00$ & 0,01 & 0,03 & 0,02 & $-0,02$ & 0,01 & 0,06 & 0,04 & 0,01 & $0,09^{*}$ & & 1,35 \\
\hline (12) FÜHRUNGSERFAHRUNG & 0,05 & 0,00 & $-0,03$ & 0,03 & $-0,01$ & $0,10^{*}$ & $0,19^{*}$ & $0,18^{*}$ & $-0,01$ & $0,10^{*}$ & $0,12^{*}$ & 1,16 \\
\hline
\end{tabular}

N=1.106.
Signifikanzniveau: $* \mathrm{p}<0,05$.

${ }_{2}^{1}$ Als Assoziationsmaße verwenden wir Cramer's V (Dummy-Variablen), Kendals-tau (ordinale Variablen) bzw. den Pearson-Korrelationskoeffizienten (metrische Variablen). Die Signifikanzniveaus sind mit dem Fisher-exact-Test berechnet.

${ }^{2} \mathrm{VIF}=$ Varianzinflationsfaktor 
Tab. 6: Koeffizienten und marginale Effekte der Probit-Modelle (abhängige Variable: ERFOLG)

\begin{tabular}{|c|c|c|c|c|c|c|c|c|}
\hline \multirow[b]{3}{*}{ Variablen } & \multicolumn{6}{|c|}{ Schätzung der Koeffizienten } & \multirow{2}{*}{\multicolumn{2}{|c|}{$\begin{array}{c}\text { Marginale Effekte } \\
\text { Modell I }\end{array}$}} \\
\hline & \multicolumn{2}{|c|}{ Modell I } & \multicolumn{2}{|c|}{ Modell II } & \multicolumn{2}{|c|}{ Modell III } & & \\
\hline & $\beta$ & $\mathbf{S F}^{1}$ & $\beta$ & $\mathrm{SF}^{1}$ & $\beta$ & $\mathrm{SF}^{1}$ & $d y / d x$ & $\mathbf{S F}^{1}$ \\
\hline \multicolumn{9}{|l|}{ Gründungsmotivation } \\
\hline NECESSITY & $-0,091$ & 0,102 & $-0,091$ & 0,102 & $-0,093$ & 0,102 & $-0,033$ & 0,036 \\
\hline ARBEITSLOSIGKEIT & $-0,053^{* * * *}$ & 0,009 & $-0,053^{* * *}$ & 0,009 & $-0,054^{* * *}$ & 0,009 & $-0,019^{* * *}$ & 0,003 \\
\hline \multicolumn{9}{|l|}{ Gründungscharakteristika } \\
\hline FREMDKAPITAL & $-0,006$ & 0,097 & $-0,006$ & 0,097 & 0,000 & 0,097 & 0,002 & 0,035 \\
\hline FRANCHISE & $-0,704^{* *}$ & 0,263 & $-0,704^{* *}$ & 0,263 & $-0,716^{* *}$ & 0,269 & $-0,273^{* *}$ & 0,108 \\
\hline TEAM & $-0,087$ & 0,124 & $-0,087$ & 0,124 & $-0,076$ & 0,124 & $-0,032$ & 0,046 \\
\hline \multicolumn{9}{|l|}{ Demographische Merkmale } \\
\hline ALTER & $-0,021^{* * *}$ & 0,006 & $-0,025$ & 0,045 & & & $-0,008^{* * *}$ & 0,002 \\
\hline ALTER $^{2}$ & & & $-0,000$ & 0,001 & & & & \\
\hline ALTERSKLASSE 26-35 & & & & & $-0,490$ & 0,342 & & \\
\hline ALTERSKLASSE 36-45 & & & & & $-0,724^{*}$ & 0,336 & & \\
\hline ALTERSKLASSE 46-55 & & & & & $-0,923^{* *}$ & 0,343 & & \\
\hline ALTERSKLASSE $\geq 56$ & & & & & $-0,994^{*}$ & 0,386 & & \\
\hline GESCHLECHT & $0,463^{* * *}$ & 0,096 & $0,463^{* * *}$ & 0,096 & $0,458^{* * *}$ & 0,096 & $-0,172^{* * *}$ & 0,036 \\
\hline DEUTSCH & 0,057 & 0,198 & 0,057 & 0,198 & 0,067 & 0,197 & $-0,021$ & 0,073 \\
\hline \multicolumn{9}{|l|}{ Humankapital } \\
\hline BRANCHENERFAHRUNG & $0,317^{* *}$ & 0,103 & $0,317^{* *}$ & 0,103 & $0,309^{* *}$ & 0,103 & $0,118^{* *}$ & 0,039 \\
\hline GRÜNDUNGSERFAHRUNG & $-0,060$ & 0,090 & $-0,060$ & 0,090 & $-0,057$ & $-0,090$ & $-0,011$ & 0,042 \\
\hline FÜHRUNGSERFAHRUNG & 0,073 & 0,094 & 0,074 & 0,094 & 0,073 & 0,094 & 0,027 & 0,034 \\
\hline BILDUNG & \multicolumn{2}{|c|}{6 Kat. $(p=0,369)$} & \multicolumn{2}{|c|}{6 Kat. $(p=0,367)$} & \multicolumn{2}{|c|}{6 Kat. $(p=0,391)$} & & \\
\hline Förderungsinstrumente & \multicolumn{2}{|c|}{3 Kat. $(p=0,803)$} & \multicolumn{2}{|c|}{3 Kat. $(p=0,803)$} & \multicolumn{2}{|c|}{3 Kat. $(p=0,787)$} & & \\
\hline Region & \multicolumn{2}{|c|}{9 Kat. $(p=0,183)$} & \multicolumn{2}{|c|}{9 Kat. $(p=0,184)$} & \multicolumn{2}{|c|}{9 Kat. $(p=0,177)$} & & \\
\hline Branchen & \multicolumn{2}{|c|}{11 Kat. $(p=0,064)$} & \multicolumn{2}{|c|}{11 Kat. $(p=0,065)$} & \multicolumn{2}{|c|}{11 Kat. $(p=0,066)$} & & \\
\hline Gründungsjahr & \multicolumn{2}{|c|}{3 Kat. $(p=0,002)$} & \multicolumn{2}{|c|}{3 Kat. $(p=0,002)$} & \multicolumn{2}{|c|}{3 Kat. $(p=0,002)$} & & \\
\hline Konstante & 0,508 & 0,591 & 0,579 & 1,032 & $0,963^{+}$ & 0,560 & & \\
\hline Pseudo $\mathrm{R}^{2}$ & \multicolumn{2}{|c|}{0,146} & \multicolumn{2}{|c|}{0,146} & \multicolumn{2}{|c|}{0,147} & & \\
\hline Log-Likelihood & -61 & & -61 & & -61 & & & \\
\hline Richtig klassifiziert & 67 & & 67 & & & & & \\
\hline Wald $\chi^{2}$ (Freiheitsgrade) & 203,15 & 3) $)^{* * * *}$ & 203,77 & t) ${ }^{* * * *}$ & 206,1 & $6)^{* * * *}$ & & \\
\hline
\end{tabular}


Tab. 7: Koeffizienten des Heckman-Modells

\begin{tabular}{|c|c|c|c|c|}
\hline \multirow[b]{2}{*}{ Variablen } & \multicolumn{2}{|c|}{ Erfolgsgleichung } & \multicolumn{2}{|c|}{ Selektionsgleichung } \\
\hline & $\beta$ & $\mathrm{SF}^{1}$ & $\beta$ & $\mathrm{SF}^{1}$ \\
\hline \multicolumn{5}{|l|}{ Gründungsmotivation } \\
\hline NECESSITY & $-0,133$ & 0,098 & $-0,337^{+}$ & 0,173 \\
\hline ARBEITSLOSIGKEIT & $-0,052^{* * *}$ & 0,008 & $-0,017$ & 0,012 \\
\hline \multicolumn{5}{|l|}{ Gründungscharakteristika } \\
\hline FREMDKAPITAL & $-0,020$ & 0,092 & 0,003 & 0,148 \\
\hline FRANCHISE & $-0,732^{* *}$ & 0,250 & $-0,504$ & 0,346 \\
\hline TEAM & $-0,158$ & 0,116 & $-0,247$ & 0,180 \\
\hline \multicolumn{5}{|l|}{ Demographische Merkmale } \\
\hline ALTER & $-0,025^{* * *}$ & 0,006 & $-0,013$ & 0,009 \\
\hline GESCHLECHT & $0,457^{* * *}$ & 0,093 & 0,004 & 0,147 \\
\hline DEUTSCH & $-0,024$ & 0,191 & $-0,288$ & 0,382 \\
\hline \multicolumn{5}{|l|}{ Humankapital } \\
\hline BRANCHENERFAHRUNG & $0,338^{* * *}$ & 0,096 & 0,127 & 0,151 \\
\hline GRÜNDUNGSERFAHRUNG & $-0,022$ & 0,087 & 0,213 & 0,139 \\
\hline FÜHRUNGSERFAHRUNG & 0,025 & 0,089 & $-0,219$ & 0,142 \\
\hline BILDUNG & \multicolumn{2}{|c|}{6 Kat. $(p=0,179)$} & \multicolumn{2}{|c|}{6 Kat. $(p=0,283)$} \\
\hline \multicolumn{5}{|l|}{ Gründungsvorbereitung } \\
\hline BUSINESSPLANUMFANG & & & $0,089^{*}$ & 0,041 \\
\hline BERATUNGSUNTERSTÜTZUNG & & & $-0,085$ & 0,152 \\
\hline Förderungsinstrumente & \multicolumn{2}{|c|}{3 Kat. $(p=0,762)$} & \multicolumn{2}{|c|}{3 Kat. $(p=0,061)$} \\
\hline Region & \multicolumn{2}{|c|}{9 Kat. $(p=0,084)$} & \multicolumn{2}{|c|}{9 Kat. $(p=0,579)$} \\
\hline Branchen & \multicolumn{2}{|c|}{11 Kat. $(p=0,399)$} & \multicolumn{2}{|c|}{11 Kat. $(p=0,084)$} \\
\hline Gründungsjahr & \multicolumn{2}{|c|}{3 Kat. $(p=0,095)$} & \multicolumn{2}{|c|}{3 Kat. $(p=0,001)$} \\
\hline Konstante & $0,966^{*}$ & 0,458 & $1,828^{*}$ & 0,757 \\
\hline $\mathrm{N}$ & \multicolumn{2}{|c|}{1106} & \multicolumn{2}{|c|}{1196} \\
\hline$\rho$ & \multicolumn{4}{|c|}{0,895} \\
\hline LR Test $(\rho=0)$ & \multicolumn{4}{|c|}{$\mathrm{p}=0,069$} \\
\hline Wald $\chi^{2}$ (Freiheitsgrade) & \multicolumn{4}{|c|}{$188,75(43)^{* * *}$} \\
\hline
\end{tabular}

